\title{
Distribution of the Error Term for the Number of Lattice Points Inside a Shifted Circle
}

\author{
Pavel M. Bleher ${ }^{1 \star}$, Zheming Cheng ${ }^{2}$, Freeman J. Dyson ${ }^{1}$, and Joel L. Lebowitz ${ }^{2,3}$ \\ 1 School of Natural Sciences, Institute for Advanced Study, Princeton, NJ 08540, USA \\ 2 Department of Mathematics, Rutgers University, New Brunswick, NJ 08903, USA \\ 3 Department of Physics, Rutgers University, New Brunswick, NJ 08903, USA
}

Received February 24, 1992; in revised form November 3, 1992

\begin{abstract}
We investigate the fluctuations in $N_{\alpha}(R)$, the number of lattice points $n \in \mathbf{Z}^{2}$ inside a circle of radius $R$ centered at a fixed point $\alpha \in[0,1)^{2}$. Assuming that $R$ is smoothly (e.g., uniformly) distributed on a segment $0 \leqq R \leqq T$, we prove that the random variable $\frac{N_{\alpha}(R)-\pi R^{2}}{\sqrt{R}}$ has a limit distribution as $T \rightarrow \infty$ (independent of the distribution of $R$ ), which is absolutely continuous with respect to Lebesgue measure. The density $p_{\alpha}(x)$ is an entire function of $x$ which decays, for real $x$, faster than $\exp \left(-|x|^{4-\varepsilon}\right)$. We also obtain a lower bound on the distribution function $P_{\alpha}(x)=\int_{-\infty}^{x} p_{\alpha}(y) d y$ which shows that $P_{\alpha}(-x)$ and $1-P_{\alpha}(x)$ decay when $x \rightarrow \infty$ not faster than $\exp \left(-x^{4+\varepsilon}\right)$. Numerical studies show that the profile of the density $p_{\alpha}(x)$ can be very different for different $\alpha$. For instance, it can be both unimodal and bimodal. We show that $\int_{-\infty}^{\infty} x p_{\alpha}(x) d x=0$, and the variance $D_{\alpha}=\int_{-\infty}^{\infty} x^{2} p_{\alpha}(x) d x$ depends continuously on $\alpha$. However, the partial derivatives of $D_{\alpha}$ are infinite at every rational point $\alpha \in \mathbf{Q}^{2}$, so $D_{\alpha}$ is analytic nowhere.
\end{abstract}

\section{Contents}

I. Introduction . . . . . . . . . . . . . . . . . . . . . . . . . 434

II. Ergodic Theorem . . . . . . . . . . . . . . . . . . . . . . . 443

III. Almost Periodicity of the Error Function . . . . . . . . . . . . 445

IV. Upper Bound on the Error Term Distribution Density . . . . . . . 450

V. Lower Bound on the Error Term Distribution Function . . . . . . 454

Appendix A. Proof of Theorem 4.1 . . . . . . . . . . . . . 456

Appendix B. Proof of Theorem 4.3 . . . . . . . . . . . . . . . . 460

References . . . . . . . . . . . . . . . . . . 468

\footnotetext{
* Permanent address: Raymond and Beverly Sackler Faculty of Exact Sciences, School of Mathematical Sciences, Tel Aviv University, Ramat Aviv 69978, Israel
} 


\section{Introduction}

Let $\alpha=\left(\alpha_{1}, \alpha_{2}\right)$ be a fixed point in the unit square, with $0 \leqq \alpha_{1}, \alpha_{2}<1$ and define

$$
N_{\alpha}(R)=\#\left\{n \in \mathbf{Z}^{2}|| n-\alpha \mid \leqq R\right\}
$$

as the number of integer points inside the circle of radius $R$ centered at $\alpha$. Clearly $N_{\alpha}(R)$ will grow for large $R$ as the area $\pi R^{2}$, but what can one say about the nature of the fluctuations about this mean value?

The classical circle problem which goes back to Gauss is to estimate the error term when $\alpha$ is the origin. The best result in this direction:

$$
\left|N_{0}(R)-\pi R^{2}\right| \leqq C_{\varepsilon} R^{(7 / 11)+\varepsilon}
$$

for every $\varepsilon>0$ was obtained by Iwaniec and Mozzochi (see [IM]). ${ }^{1}$

Hardy's careful conjecture (see [H1]): "it is not unlikely that

$$
\left|N_{0}(R)-\pi R^{2}\right|=O\left(R^{(1 / 2)+\varepsilon}\right)
$$

for all positive $\varepsilon, "$ remains open. In Table 1 and Fig. 1 we present numerical results. They are consistent with the asymptotics:

$$
\begin{aligned}
& \min _{r \leqq R}\left\{N_{0}(r)-\pi r^{2}\right\} \sim-\text { const. } R^{1 / 2}(\log R)^{\lambda_{-}}, \\
& \max _{r \leqq R}\left\{N_{0}(r)-\pi r^{2}\right\} \sim \text { const. } R^{1 / 2}(\log R)^{\lambda_{+}}
\end{aligned}
$$

with $\lambda_{-}=0.6 \pm 0.1$ and $\lambda_{+}=0.3 \pm 0.1$. This should be compared with the $\Omega$-results of Hardy (see [H2]),

$$
\lim _{R \rightarrow \infty} \inf \frac{N_{0}(R)-\pi R^{2}}{R^{1 / 2}(\log R)^{1 / 4}}<0,
$$

and

$$
\lim _{R \rightarrow \infty} \sup \frac{N_{0}(R)-\pi R^{2}}{R^{1 / 2}}>0 .
$$

Later, $\log \log$-improvements of these estimates were obtained (for the best results in this direction see [Haf] and [CK], and for a review, the monograph [G]), however, there has been no improvement in the exponents $1 / 4$ and 0 at the log-term.

In this paper we shall be interested in the limit distribution of the suitably normalized error term

$$
F_{\alpha}(R)=\frac{N_{\alpha}(R)-\pi R^{2}}{\sqrt{R}}
$$

assuming that $R$ is uniformly (or approximately so) distributed on $[0, T]$ and $T \rightarrow \infty$. Our work follows closely and generalizes recent results of Heath-Brown who proved in [H-B] the existence of the limit distribution of $F_{0}(R)$, assuming that $R$ is uniformly distributed on $[0, T]$. Heath-Brown also showed that this limit

\footnotetext{
1 Very recently Huxley obtained a stronger result, improving the exponent 7/11 to 46/73 (see [Hux])
} 
Table 1a, b. Minima and maxima of $\left\{F_{0}(r) \mid r<R\right\}$

\begin{tabular}{ll}
\hline (a) $R^{2}$ & $\min \left\{\left(N_{0}(r)-\pi r^{2}\right) / r^{1 / 2} \mid r<R\right\}$ \\
\hline 1 & -0.854372 \\
4 & -1.006055 \\
16 & -1.050312 \\
25 & -1.702022 \\
144 & -1.772309 \\
288 & -1.915399 \\
1681 & -1.994813 \\
3025 & -2.061242 \\
3961 & -2.154738 \\
5184 & -2.304541 \\
31681 & -2.386141 \\
38018 & -2.516160 \\
40321 & -2.566369 \\
106250 & -2.590165 \\
114242 & -2.621929 \\
114244 & -2.671462 \\
201601 & -2.847067 \\
574561 & -2.950457 \\
1149109 & -3.014438 \\
1149122 & -3.122152 \\
7441954 & -3.231051 \\
40589629 & -3.235172 \\
40589636 & -3.265116 \\
40589641 & -3.303641 \\
133055698 & -3.307234 \\
133055701 & -3.312526 \\
133055714 & -3.345365 \\
133055721 & -3.397335 \\
133055744 & -3.606301 \\
133055753 & -3.622178 \\
133055761 & -3.656102 \\
133055770 & -3.731411 \\
\hline &
\end{tabular}

\begin{tabular}{ll}
\hline (b) $R^{2}$ & $\max \left\{\left(N_{0}(r)-\pi r^{2}\right) / r^{1 / 2} \mid r<R\right\}$ \\
\hline 1 & 0.741397 \\
2 & 0.911407 \\
5 & 1.411856 \\
53 & 1.551844 \\
340 & 1.566243 \\
586 & 1.623847 \\
850 & 1.673214 \\
986 & 1.949967 \\
5525 & 2.022154 \\
17225 & 2.056893 \\
147652 & 2.087299 \\
457317 & 2.105906 \\
574930 & 2.160667 \\
776533 & 2.185626 \\
3710538 & 2.200426 \\
3823300 & 2.253751 \\
3823301 & 2.297583 \\
\hline
\end{tabular}


Table 1a, b. (Continued)

\begin{tabular}{ll}
\hline (a) $R^{2}$ & $\min \left\{\left(N_{0}(r)-\pi r^{2}\right) / r^{1 / 2} \mid r<R\right\}$ \\
\hline 3823306 & 2.300217 \\
7646605 & 2.328358 \\
7646609 & 2.415100 \\
34307377 & 2.447120 \\
34307380 & 2.481394 \\
34307381 & 2.506720 \\
5792465045 & 2.512855 \\
34788691946 & 2.517548 \\
34788692025 & 2.532156 \\
34788692045 & 2.533235 \\
35756257745 & 2.582907 \\
\hline
\end{tabular}

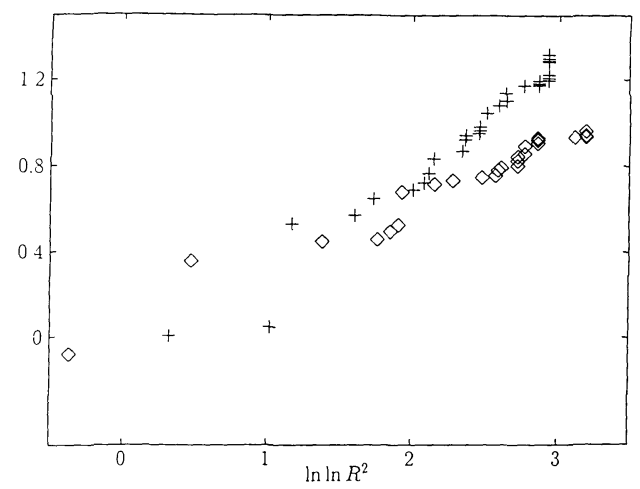

Fig. 1. Plots of $\ln \left(-\min \left\{F_{0}(r) \mid r<R\right\}\right)(+)$ and of $\ln \left(\max \left\{F_{0}(r) \mid r<R\right\}\right)(\diamond)$ vs. $\ln \ln R^{2}$.

distribution is absolutely continuous with respect to Lebesgue measure and its distribution density $p_{0}(x)$ is an entire function which decreases on the real line faster than polynomially.

This probabilistic approach to problems in number theory fits in well with current interest in problems relating to the statistics of eigenvalues of "typical" Schrödinger operators. The numbers $(n-\alpha)^{2}$, whose distribution we study here, may be thought of as eigenvalues of the "displaced" Laplace operator $(i \nabla-\alpha)^{2}$ on the two dimensional torus corresponding to the classically integrable Hamiltonian $(p-\alpha)^{2}($ see $[\mathrm{B}, \mathrm{CCG}])$. More generally it was shown by Colin de Verdiere [CdV] (see also the works of Sinai [S] and Bleher [B11]) that the asymptotics of eigenvalues of the Laplacian on surfaces of revolution may be related to the type of statistics we study here with the circle replaced by a suitable curve.

An early study of $F_{\alpha}(R)$ when $\alpha$ itself is a random variable uniformly distributed on $[0,1)^{2}$ was carried out by Kendall $[\mathrm{K}]$. He showed that the average second moment

$$
\lim _{T \rightarrow \infty} \frac{1}{T} \int_{0}^{T} d x \int_{0 \leqq \alpha_{1}, \alpha_{2} \leqq 1} d \alpha\left(F_{\alpha}(\sqrt{x})\right)^{2}=a^{2}>0
$$


exists and can be computed explicitly, $a=0.67649 \ldots$. Similar results hold for more general oval figures (see $[\mathrm{K}]$ ).

In the present work we do not consider averages over $\alpha$. Instead we use Heath-Brown's methods to prove the existence of the limit distribution of $F_{\alpha}(R)$ for all $\alpha \in[0,1)^{2}$. The limit distribution is absolutely continuous and its density decreases at infinity roughly as $\exp \left(-\right.$ const. $\left.|x|^{4}\right)$. This implies that there is no central limit theorem for the fluctuations. We also show that this limit distribution is the same for a class of distributions of $R$ described by a density $\rho(R / T) d R / T$, where $\rho(x)$ is an integrable non-negative function on $[0,1]$ with $\int_{0}^{1} \rho(x) d x=1$.

In contrast with the above results, Beck (see $[\mathrm{Be}]$ ) recently derived different behavior for some very irrationally oriented rectangles. A natural parameter of the orientation of the rectangle is $\eta=\tan \varphi$, the tangent of the angle between $x$-axis and a side of the rectangle. Hardy and Littlewood, Ostrowsky and others showed that the behavior of the error term as $R \rightarrow \infty$, for a rectangle of orientation $\eta$ and of sides $R a_{1}, R a_{2}$, depends on the behavior of the coefficients in the expansion of $\eta$ into a continued fraction. Assuming that the continued fraction coefficients are bounded, Beck proved in $[\mathrm{Be}]$ that for such a rectangle with the center at any fixed point $\alpha$ in a plane, the error term, after normalization by $\sqrt{\log R}$, satisfies a central limit theorem. In this case (i.e., when the continued fraction coefficients are bounded) a classical theorem proved by Littlewood and Hardy, and independently by Ostrowsky gives a bound $C \log R$ for the error term.

Before giving a precise formulation of our main results, we present numerical data of the distribution of $F_{\alpha}(R)$ for several values of $\alpha$. In Fig. 2 we plot the density $p_{0}(x)$ of the random variable $F_{0}(R)$ for $R^{2}$ uniformly distributed in the range $\left[9 \times 10^{8}, 49 \times 10^{8}\right]$. This corresponds to $\rho(x)=2 x$. The maximum of $p_{0}(x)$ occurs at positive $x$ while its tail is skewed towards negative $x$. In fact a computation of the third moment of $F_{0}$ shows it to be negative (see the work of Tsang [Ts]). Figures 3 , 4 show $p_{\alpha}(x)$ for $\alpha=(0.3437 \ldots, 0.4304 \ldots)$ and $\alpha=(0.4685 \ldots, 0.01814 \ldots)$, respectively. In Fig. 5 we plot the average of $p_{\alpha}(x)$ over $\alpha$, corresponding to the case studied by Kendall $[\mathrm{K}]$. The shape of $p_{\alpha}$ varies greatly as $\alpha$ changes.

The width of the distribution is largest for $\alpha=0$ in Figs. 2-4. This can be understood from the equation (1.20) below for the variance $D_{\alpha}$. It is clear that $D_{\alpha}$ has a maximum at $\alpha=0$ and at $\alpha=(1 / 2,1 / 2)$. It is also easy to see that $D_{\alpha}$ is continuous in $\alpha$. A more detailed analysis of (1.20), which will be described elsewhere, shows that $D_{\alpha}$ is quite wild: when $\alpha \rightarrow \beta$, where $\beta$ is a point with rational coordinates,

$$
D_{\alpha}=D_{\beta}-C_{\beta}|\alpha-\beta||\log | \alpha-\beta||+o(|\alpha-\beta||\log | \alpha-\beta||),
$$

with some $C_{\beta}>0$. This shows that $D_{\alpha}$ has a sharp local maximum with infinite partial derivatives at every rational point.

Our results do not imply the existence of higher moments of $F_{\alpha}(R)$, as $T \rightarrow \infty$, i.e., we do not know if

$$
\lim _{T \rightarrow \infty} \frac{1}{T} \int_{0}^{T}\left(F_{\alpha}(R)\right)^{k} \rho(R / T) d R
$$

exists for $k>2$, and if it does exist, whether it is equal to $\int_{-\infty}^{\infty} x^{k} p_{\alpha}(x) d x$. For $\alpha=0$ Heath-Brown showed the existence of the moments for $k \leqq 9$. It seems likely that similar results hold for all $\alpha$. 


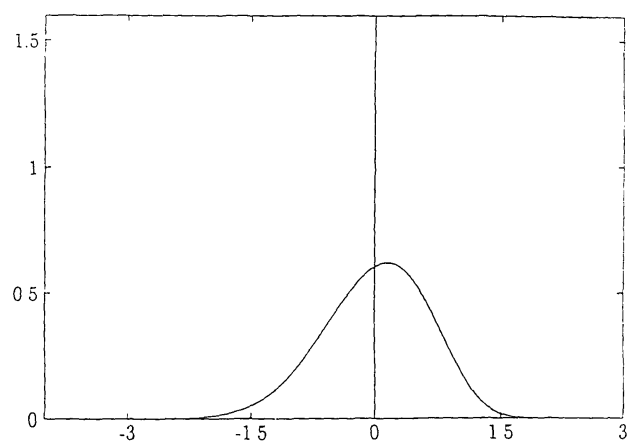

Fig. 2. Plots of $\bar{p}_{\alpha}(x)=(2 \pi)^{1 / 2} p_{\alpha}\left((2 \pi)^{1 / 2} x\right)$ vs. $x$ for: $\alpha=0$

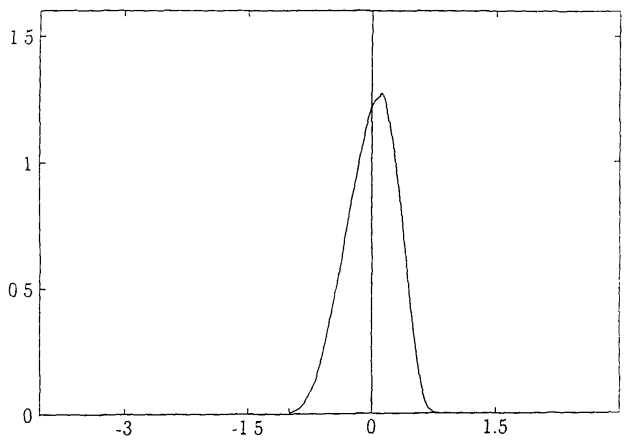

Fig. 3. Plots of $\bar{p}_{\alpha}(x)=(2 \pi)^{1 / 2} p_{\alpha}\left((2 \pi)^{1 / 2} x\right)$ vs. $x$ for: $\alpha=(0.3437 \ldots, 0.4304 \ldots)$

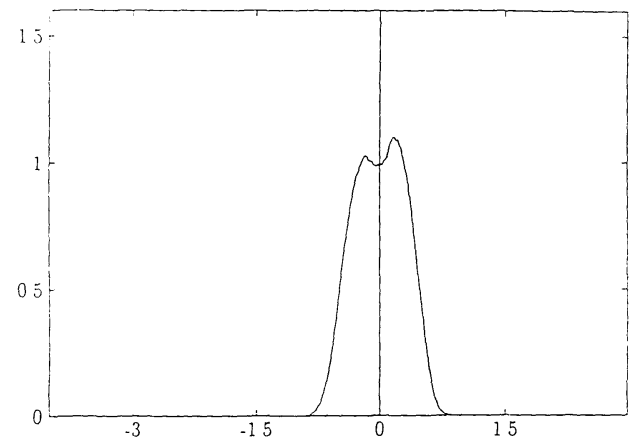

Fig. 4. Plots of $\bar{p}_{\alpha}(x)=(2 \pi)^{1 / 2} p_{\alpha}\left((2 \pi)^{1 / 2} x\right)$ vs. $x$ for: $\alpha=(0.4658 \ldots, 0.01814 \ldots)$

\section{Statement of Results.}

Let $\rho(x) \geqq 0$ be an arbitrary probability density on $[0,1]$. The reader may think of $\rho(x)$ as equal to 1 or $2 x$, corresponding to uniform distribution of $R$ or $R^{2}$ respectively. 


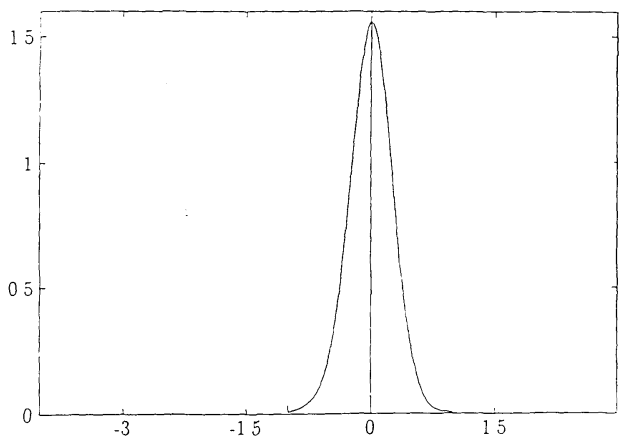

Fig. 5. Plots of $\bar{p}_{\alpha}(x)=(2 \pi)^{1 / 2} p_{\alpha}\left((2 \pi)^{1 / 2} x\right)$ vs. $x$ for: average of $p_{\alpha}(x)$ over $\alpha \in[0,1)^{2}$

Theorem 1.1. For every piecewise continuous bounded function $g(x)$ on $\mathbf{R}^{1}$,

$$
\lim _{T \rightarrow \infty} \frac{1}{T} \int_{0}^{T} g\left(F_{\alpha}(R)\right) \rho(R / T) d R=\int_{-\infty}^{\infty} g(x) p_{\alpha}(x) d x,
$$

where $p_{\alpha}(x)$ is a probability density on $\mathbf{R}^{1}$ which can be extended to the whole complex plane as an entire function of $x$. For real $x, p_{\alpha}(x)$ satisfies for every $\varepsilon>0$ and $|x|>x_{0}(\varepsilon, \alpha)$ the upper bound,

$$
0 \leqq p_{\alpha}(x)<\exp \left(-|x|^{4-\varepsilon}\right)
$$

The distribution function $P_{\alpha}(x)=\int_{-\infty}^{x} p_{\alpha}\left(x^{\prime}\right) d x^{\prime}$ satisfies for every $\varepsilon>0$ and $x>x_{0}(\varepsilon, \alpha)$ the lower bound,

$$
P_{\alpha}(-x), \quad 1-P_{\alpha}(x)>\exp \left(-x^{4+\varepsilon}\right) .
$$

This theorem expresses the weak convergence of the distribution of $F_{\alpha}(R)$ to an absolutely continuous distribution $p_{\alpha}(x) d x$ when $T \rightarrow \infty$, and gives bounds on the behavior of $p_{\alpha}(x)$ at infinity. Recently Bleher proved the existence of a weak limit of the distribution of $F_{\alpha}(R)$ for any smooth strictly convex oval enclosing the origin (see [B12]). He proved also (1.3), (1.4) for ovals satisfying a condition of absence of resonances (see [B13]).

The techniques used to analyse the behavior of $N_{\alpha}(R)$ are all based on the Poisson summation formula

$$
\sum_{n \in \mathbf{Z}^{2}} f(n)=\sum_{n \in \mathbf{Z}^{2}} \tilde{f}(2 \pi n)
$$

where $\tilde{f}$ is the two dimensional Fourier transform of $f$,

$$
\tilde{f}(\xi)=\int_{\mathbf{R}^{2}} e^{i x \xi} f(x) d x
$$

and $x \xi$ is the usual scalar product in $\mathbf{R}^{2}, x \xi=x_{1} \xi_{1}+x_{2} \xi_{2}$. Defining, for $x \in \mathbf{R}^{2}$,

we find immediately

$$
\chi_{\alpha}(x ; R)=\begin{aligned}
& 1 \text { if }|x-\alpha| \leqq R \\
& 0 \text { if }|x-\alpha|>R,
\end{aligned}
$$

$$
N_{\alpha}(R)=\sum_{n \in \mathbf{Z}^{2}} \chi_{\alpha}(n ; R)=\sum_{n \in \mathbf{Z}^{2}} \tilde{\chi}_{\alpha}(2 \pi n ; R),
$$


where

$$
\begin{aligned}
\tilde{\chi}_{\alpha}(\xi ; R) & =\int_{\mathbf{R}^{2}} e^{i x \xi} \chi_{\alpha}(x ; R) d x=\int_{|x-\alpha| \leqq R} e^{i x \xi} d x \\
& =e^{i \xi \alpha} \int_{|x| \leqq R} e^{i x \xi} d x=e^{i \xi \alpha} \frac{2 \pi R}{|\xi|} J_{1}(R|\xi|)
\end{aligned}
$$

and $J_{1}(t)$ is the Bessel function. Noting that

$$
\tilde{\chi}_{\alpha}(0 ; R)=\int_{\mathbf{R}^{2}} \chi_{\alpha}(x ; R) d x=\int_{|x-\alpha| \leqq R} d x=\pi R^{2}
$$

yields

$$
N_{\alpha}(R)-\pi R^{2}=\sum_{n \neq 0} \tilde{\chi}_{\alpha}(2 \pi n ; R)=\sum_{n \neq 0} e(n \alpha) \frac{J_{1}(2 \pi|n| R)}{|n|} R,
$$

which is a variant of the Hardy-Voronoi summation formula (see also the work [K] where various generalizations of (1.9) are discussed). We use here and later on the notation

$$
e(t)=e^{2 \pi i t}
$$

The well known relation

$$
J_{1}(t)=\sqrt{\frac{2}{\pi|t|}} \cos \left(|t|-\frac{3 \pi}{4}\right)+O\left(|t|^{-3 / 2}\right),
$$

then gives

$$
\tilde{\chi}_{\alpha}(2 \pi n ; R)=\pi^{-1} \sqrt{R} \frac{e(n \alpha)}{|n|^{3 / 2}} \cos \left(2 \pi|n| R-\frac{3 \pi}{4}\right)+O\left(|n|^{-5 / 2} R^{-1 / 2}\right)
$$

so that

$$
N_{\alpha}(R)-\pi R^{2}=\pi^{-1} \sqrt{R} \sum_{n \neq 0} \frac{e(n \alpha)}{|n|^{3 / 2}} \cos \left(2 \pi|n| R-\frac{3 \pi}{4}\right)+O\left(R^{-1 / 2}\right) .
$$

This leads us to the formula

$$
\begin{aligned}
F_{\alpha}(R) & =\frac{N_{\alpha}(R)-\pi R^{2}}{\sqrt{R}}=\sum_{n \neq 0} \frac{\tilde{\chi}_{\alpha}(2 \pi n ; R)}{\sqrt{R}} \\
& =\pi^{-1} \sum_{n \neq 0} \frac{e(n \alpha)}{|n|^{3 / 2}} \cos \left(2 \pi|n| R-\frac{3 \pi}{4}\right)+O\left(R^{-1}\right) .
\end{aligned}
$$

The basic idea of Heath-Brown is to rewrite (1.12) as a sum of terms representing a flow on an infinite-dimensional torus with incommensurate frequencies: $R$ playing the role of time. The problem of finding a limiting distribution is then a problem in ergodic theory.

To do this we first group terms in (1.12) with commensurate frequencies, i.e., all those $n \in \mathbf{Z}^{2}$ for which $|n|=k \sqrt{m}, k=1,2, \ldots$, where $m>0$ is a fixed square-free natural number. Let us recall that $m \in \mathbf{N}$ is square free if $m \neq k^{2} l$ with $k>1 ; k, l \in \mathbf{N}$. 
We define for every square free $m$ a function

$$
\begin{aligned}
f_{m}(t ; \alpha) & =\pi^{-1} \sum_{k=1}^{\infty} \sum_{n:|n|=k \sqrt{m}} \frac{e(n \alpha)}{|n|^{3 / 2}} \cos \left(2 \pi k t-\frac{3 \pi}{4}\right) \\
& =\frac{\pi^{-1}}{m^{3 / 4}} \sum_{k=1}^{\infty} \frac{1}{k^{3 / 2}} \cos (2 \pi k t-3 \pi / 4) \sum_{n:|n|=k \sqrt{m}} e(n \alpha),
\end{aligned}
$$

which is periodic in $t$ with period 1 (for $m$ not square free we define $f_{m}(t ; \alpha)=0$ ). Then (1.12) can be written as

$$
F_{\alpha}(R)=\sum_{\text {square free } m} f_{m}(\sqrt{m} R ; \alpha)+O\left(R^{-1}\right) .
$$

As is well known, the $\sqrt{m}$ with square-free $m$ are linearly independent over the field of rational numbers $\mathbf{Q}$. We are thus led to the study of limit distributions of almost periodic functions of the form

$$
F(t)=\sum_{n=1}^{\infty} a_{n}\left(\gamma_{n} t\right)
$$

with $\gamma_{n}$ linearly independent over $\mathbf{Q}$. If the sum were finite, say, up to some $N \geqq 1$, the Birkhoff-Khinchin ergodic theorem in the strengthened form (see, e.g. [CFS]) would imply that for every continuous bounded function $g(x)$ on a line,

$$
\lim _{T \rightarrow \infty} \frac{1}{T} \int_{0}^{T} g(F(t)) d t=\int_{0}^{1} \ldots \int_{0}^{1} g\left(\sum_{n=1}^{N} a_{n}\left(t_{n}\right)\right) d t_{1} \ldots d t_{N}=\int_{-\infty}^{\infty} g(x) v_{N}(d x),
$$

where $v_{N}(d x)$ is the distribution of the finite series $\sum_{n=1}^{N} a_{n}\left(\theta_{n}\right)$, when the $\theta_{n}$ are independent random variables uniformly distributed on $[0,1]$. This means, roughly speaking, that the functions $\gamma_{n} t \bmod 1, n=1, \ldots, N$, behave like independent random variables uniformly distributed on $[0,1)$, as $t$ varies over a sufficiently large range. Our problem is that the series in (1.14) is infinite, and in fact only conditionally convergent, since one can see from (1.13) that $f_{m}(t ; \alpha) \sim m^{-3 / 4}$. So the problem is to prove the existence of a limit distribution of conditionally convergent but square-summable series of periodic functions with incommensurable periods.

Heath-Brown has proved in [H-B] some general theorems concerning this problem. We will use a modified version of Heath-Brown's theorems to prove Theorem 1.1.

Following [H-B] we formulate conditions on the functions $F(t)$ and $a_{n}(t)$ in the form of a hypothesis.

Hypothesis $\left(H_{0}\right)$. Let $a_{1}(t), a_{2}(t), \ldots$ be continuous real valued periodic functions of period 1 such that

$$
\begin{gathered}
\int_{0}^{1} a_{n}(t) d t=0, \quad n \in \mathbf{N}, \\
\sum_{n=1}^{\infty} \int_{0}^{1} a_{n}(t)^{2} d t<\infty .
\end{gathered}
$$

Suppose that there are positive constants $\gamma_{1}, \gamma_{2}, \ldots$, which are linearly independent over $\mathbf{Q}$, such that

$$
\lim _{N \rightarrow \infty} \limsup _{T \rightarrow \infty} \frac{1}{T} \int_{0}^{T} \min \left\{1,\left|F(t)-\sum_{n \leqq N} a_{n}\left(\gamma_{n} t\right)\right|\right\} d t=0 .
$$


We prove the following ergodic theorem which will then be used to prove Theorem 1.1.

Theorem 1.2. If $F(t)$ satisfies Hypothesis $\left(H_{0}\right)$, then for every probability density $\rho(x)$ on $[0,1]$ and for every continuous bounded function $g(x)$ on $\mathbf{R}^{1}$,

$$
\lim _{T \rightarrow \infty} \frac{1}{T} \int_{0}^{T} g(F(t)) \rho(t / T) d t=\int_{-\infty}^{\infty} g(x) v(d x),
$$

where $v(d x)$ is the distribution of the random series

$$
\eta=\sum_{n=1}^{\infty} a_{n}\left(t_{n}\right)
$$

and $t_{n}$ are independent random variables uniformly distributed on $[0,1]$.

As a consequence of Theorems 1.1 and 1.2 the variance of the limit distribution $p_{\alpha}(x) d x$ is given by the formula

where

$$
D_{\alpha}=\int_{-\infty}^{\infty} x^{2} p_{\alpha}(x) d x=\left(2 \pi^{2}\right)^{-1} \sum_{m=1}^{\infty} \frac{\left|r_{\alpha}(m)\right|^{2}}{m^{3 / 2}},
$$

$$
r_{\alpha}(m)=\sum_{n \in \mathbf{Z}^{2}:|n|^{2}=m} e(n \alpha) .
$$

As was shown in [B12], $D_{\alpha}$ coincides with a squared $B^{2}$-norm of $F_{\alpha}(R)$ :

$$
D_{\alpha}=\lim _{T \rightarrow \infty} \frac{1}{T} \int_{0}^{T}\left|F_{\alpha}(R)\right|^{2} d R .
$$

The outline of the rest of the paper is as follows. In Sect. II we prove Theorem 1.2 , and in Sect. III we prove that the error function $F_{\alpha}(R)$ satisfies the conditions of this theorem, so that a limit distribution of $F_{\alpha}(R)$ exists. In Sect. IV we prove that the limit distribution is absolutely continuous, and we prove an upper bound on the distribution density. In Sect. V we prove a lower bound on the distribution function, and we conclude the proof of Theorem 1.1. In Appendix A we prove a general theorem on the analytic properties of distribution functions of random series. This theorem is used in Sect. IV. In Appendix B we prove a numbertheoretical estimate of $r_{\alpha}(m)$ which plays an essential role in proving upper and lower bounds on the limit distribution.

Many problems remain unsolved. One is the convergence of higher moments of $F_{\alpha}(R)$ to the moments of the limit distribution. Another is to extend Theorem 1.1 to higher dimensions and to more general strictly convex domains.

Convex ovals with flat points like

$$
\left(a_{11} x_{1}+a_{12} x_{2}\right)^{2 k}+\left(a_{21} x_{1}+a_{22} x_{2}\right)^{2 k}=1, \quad k>1,
$$

deserve a special study. In this case the contribution to the error term $N_{\alpha}(R)-A R^{2}$, where $A$ is the area enclosed by the curve, from a neighborhood of the flat points can change the $R^{1 / 2}$-asymptotics to a higher power of $R$. The arithmetic properties of the slope of the oval at flat points are important in this problem.

Heath-Brown proved in $[\mathrm{H}-\mathrm{B}]$ the existence and properties of limit distributions for a number of functions of interest in number theory. We conjecture that the 
lower and upper estimates of Theorem 1.1 hold for the densities of those limit distributions as well.

A more general problem is the existence and properties of limit distributions of the oscillating part in the Weyl asymptotics for the spectral function of elliptic operators on compact manifolds. In the simplest case of the operator $-(\nabla-i \alpha)^{2}$ on a two-dimensional torus, this problem reduces to the shifted circle problem studied in the present work. We believe that Theorem 1.1 actually holds in a much more general situation.

\section{Ergodic Theorem}

Proof of Theorem 1.2. We shall first prove Theorem 1.2 in the particular case $\rho(x)=1$. In addition, we shall assume first that $g(x) \in C_{0}^{\infty}\left(\mathbf{R}^{1}\right)$, i.e., $g(x)$ is an infinitely differentiable function with a compact support. We shall show that under these assumptions,

$$
\lim _{T \rightarrow \infty} \frac{1}{T} \int_{0}^{T} g(F(t)) d t=\int_{-\infty}^{\infty} g(x) v(d x)
$$

The condition $g(x) \in C_{0}^{\infty}$ implies that for all $x, y$,

$$
|g(x)-g(y)| \leqq C \min \{1,|x-y|\}
$$

with some $C>0$. So from (1.17),

$$
\frac{1}{T} \int_{0}^{T}\left|g(F(t))-g\left(F_{N}(t)\right)\right| d t \leqq C \frac{1}{T} \int_{0}^{T} \min \left\{1,\left|F(t)-F_{N}(t)\right|\right\} d t \leqq \frac{\varepsilon}{3}
$$

when $N \geqq N_{0}(\varepsilon)$ and $T \geqq T_{0}(\varepsilon, N)$, where

$$
F_{N}(t)=\sum_{n \leqq N} a_{n}\left(\gamma_{n} t\right)
$$

The function $F_{N}(t)$ is a continuous quasiperiodic function so by a strengthened variant of the Birkhoff-Khinchin ergodic theorem (see e.g., [CFS], pp. 39 and 69),

$$
\left|\frac{1}{T} \int_{0}^{T} g\left(F_{N}(t)\right) d t-\int_{0}^{1} \ldots \int_{0}^{1} g\left(\sum_{n \leqq N} a_{n}\left(t_{n}\right)\right) d t_{1} \ldots d t_{N}\right| \leqq \frac{\varepsilon}{3}
$$

when $T \geqq T_{1}(\varepsilon, N)$. Let $v_{N}(d x)$ be the distribution of

Then

$$
\eta_{N}=\sum_{n=1}^{N} a_{n}\left(t_{n}\right)
$$

and hence,

$$
\int_{0}^{1} \ldots \int_{0}^{1} g\left(\sum_{n \leqq N} a_{n}\left(t_{n}\right)\right) d t_{1} \ldots d t_{N}=\int_{-\infty}^{\infty} g(x) v_{N}(d x)
$$

$$
\left|\frac{1}{T} \int_{0}^{T} g\left(F_{N}(t)\right) d t-\int_{-\infty}^{\infty} g(x) v_{N}(d x)\right| \leqq \frac{\varepsilon}{3} .
$$

Due to $(1.15),(1.16) \eta_{N}$ converges weakly to $\eta$, so

$$
\left|\int_{-\infty}^{\infty} g(x) v_{N}(d x)-\int_{-\infty}^{\infty} g(x) v(d x)\right| \leqq \frac{\varepsilon}{3}
$$


when $N \geqq N_{1}(\varepsilon)$. Put $N=\max \left\{N_{0}(\varepsilon), N_{1}(\varepsilon)\right\}$. Then for $T \geqq \max \left\{T_{0}(\varepsilon, N)\right.$, $\left.T_{1}(\varepsilon, N)\right\}$,

$$
\left|\frac{1}{T} \int_{0}^{T} g(F(t)) d t-\int_{-\infty}^{\infty} g(x) v(d x)\right| \leqq \varepsilon
$$

so (2.1) follows.

By implication, (2.1) holds not only for $C_{0}^{\infty}$ but for all continuous bounded functions $g(x)$. Indeed, first by continuity we can extend (2.1) to the class $C_{0}$ of continuous functions with compact support. Then second, if $\psi(x) \in C_{0}^{\infty}\left(\mathbf{R}^{1}\right)$ is a function, such that $0 \leqq \psi(x) \leqq 1$ for all $x$ and $\psi(x)=1$ for $|x| \leqq 1$, then for every $\varepsilon>0$ there exists $A>0$ such that

$$
1-\varepsilon \leqq \int_{-\infty}^{\infty} \psi(x / A) v(d x) \leqq 1
$$

Hence by (2.1) there exists $T_{0}=T_{0}(\varepsilon, A)$ such that for every $T \geqq T_{0}$,

$$
1-2 \varepsilon \leqq \frac{1}{T} \int_{0}^{T} \psi(F(t) / A) d t \leqq 1 .
$$

It implies that

$$
0 \leqq \frac{1}{T} \int_{0}^{T}(1-\psi(F(t) / A)) d t \leqq 2 \varepsilon,
$$

and so for every continuous bounded function $g(x)$,

$$
\left|\frac{1}{T} \int_{0}^{T} g(F(t))(1-\psi(F(t) / A)) d t\right| \leqq 2 \varepsilon \sup _{x \in \mathbb{R}^{1}}|g(x)| .
$$

Similarly, (2.5) implies that

$$
\left|\int_{-\infty}^{\infty} g(x)(1-\psi(x / A)) v(d x)\right| \leqq \varepsilon \sup _{x \in \mathbf{R}^{1}}|g(x)| .
$$

In addition, by (2.1),

$$
\left|\frac{1}{T} \int_{0}^{T} g(F(t)) \psi(F(t) / A) d t-\int_{-\infty}^{\infty} g(x) \psi(x / A) v(d x)\right| \leqq \varepsilon
$$

when $T \geqq T_{1}(\varepsilon, A)$. Therefore

$$
\left|\frac{1}{T} \int_{0}^{T} g(F(t)) d t-\int_{-\infty}^{\infty} g(x) v(d x)\right| \leqq \varepsilon\left(1+3 \sup _{x \in \mathbf{R}^{1}}|g(x)|\right),
$$

when $T \geqq \max \left\{T_{0}(\varepsilon, A), T_{1}(\varepsilon, A)\right\}$, which proves $(2.1)$ for every bounded continuous function $g(x)$. Thus for the case $\rho(x)=1$ Theorem 1.2 is proved.

Assume now that $\rho(x)$ is a step-wise function consisting of a finite number of steps. Because of the linearity of formula (1.18), it is enough to prove (1.18) for the function

$$
\rho(x)=\frac{1}{b-a} \chi_{[a, b]}(x)
$$


consisting of a single step. In the single step case, due to (2.1),

$$
\begin{aligned}
& \frac{1}{T} \int_{0}^{T} g(F(t)) \rho(t / T) d t=\frac{1}{T(b-a)} \int_{T a}^{T b} g(F(T)) d t \\
& \quad=\frac{1}{b-a}\left(b \frac{1}{T b} \int_{0}^{T b} g(F(t)) d t-a \frac{1}{T a} \int_{0}^{T a} g(F(t)) d t\right) \\
& \quad \rightarrow{ }_{T \rightarrow \infty} \frac{1}{b-a}\left(b \int_{-\infty}^{\infty} g(x) v(d x)-a \int_{-\infty}^{\infty} g(x) v(d x)\right)=\int_{-\infty}^{\infty} g(x) v(d x)
\end{aligned}
$$

as stated in (1.18), above.

The general case follows now by approximating $\rho(x) \in L^{1}([0,1])$ by step-wise functions $\rho_{\varepsilon}(x)$ with

$$
\int_{0}^{1}\left|\rho_{\varepsilon}(x)-\rho(x)\right| d x<\varepsilon, \quad \varepsilon>0
$$

\section{Almost Periodicity of the Error Function}

In this section we will prove that Hypothesis $\left(H_{0}\right)$ holds for the error function $F_{\alpha}(R)$. To that end we will prove the following theorem:

\section{Theorem 3.1.}

$$
\lim _{N \rightarrow \infty} \lim _{T \rightarrow \infty} \sup \frac{1}{T} \int_{0}^{T}\left|F_{\alpha}(R)-\sum_{m \leqq N} f_{m}(\sqrt{m} R ; \alpha)\right| d R=0 .
$$

Proof. Define a $\delta$-shaped Gaussian function of $x \in \mathbf{R}^{2}$,

$$
\lambda(x ; T)=\pi T^{2} \exp \left(-\pi^{2} T^{2}|x|^{2}\right),
$$

so that $\int_{\mathbf{R}^{2}} \lambda(x ; T) d x=1$. Define then the smoothed characteristic function

$$
\chi_{\alpha}(x ; R, T)=\chi_{\alpha}(\cdot ; R) * \lambda(\cdot ; T)=\int_{\mathbf{R}^{2}} \chi_{\alpha}(y ; R) \lambda(x-y ; T) d y
$$

and the functions

$$
N_{\alpha}(R ; T)=\sum_{n \in \mathbf{Z}^{2}} \chi_{\alpha}(n ; R, T)
$$

and

$$
F_{\alpha}(R ; T)=\frac{N_{\alpha}(R ; T)-\pi R^{2}}{\sqrt{R}}
$$

Define also

$$
f_{m}(t ; \alpha, T)=\frac{\pi^{-1}}{m^{3 / 4}} \sum_{k=1}^{\infty} \frac{\exp \left(-m k^{2} / T^{2}\right)}{k^{3 / 2}} \cos (2 \pi k t-3 \pi / 4) \sum_{n:|n|=k \sqrt{m}} e(n a)
$$

when $m$ is square free, and $f_{m}(t ; \alpha, T)=0$ otherwise.

Proof of Theorem 3.1 is based on three lemmas: 


\section{Lemma 3.2.}

$$
\frac{1}{T} \int_{0}^{T}\left|F_{\alpha}(R ; T)-F_{\alpha}(R)\right| d R \leqq C T^{-1 / 2},
$$

where $C>0$ is an absolute constant.

\section{Lemma 3.3.}

$$
\frac{1}{T} \int_{0}^{T}\left|F_{\alpha}(R ; T)-\sum_{m \leqq N} f_{m}(\sqrt{m} R ; \alpha, T)\right|^{2} d R \leqq C\left(T^{-1 / 2}+N^{-1 / 3}\right),
$$

where $C>0$ is an absolute constant.

Lemma 3.4. If $T \geqq N^{1 / 2}$, then

$$
\frac{1}{T} \int_{0}^{T} \sum_{m \leqq N}\left|f_{m}(\sqrt{m} R ; \alpha, T)-f_{m}(\sqrt{m} R ; \alpha)\right| d R \leqq C N^{1 / 2} T^{-0.48},
$$

where $C>0$ is an absolute constant.

Assume $T>N$. Then the Schwarz inequality and Lemma $3.3 \mathrm{imply}$ that,

$$
\begin{aligned}
& \frac{1}{T} \int_{0}^{T}\left|F_{\alpha}(R ; T)-\sum_{m \leqq N} f_{m}(\sqrt{m} R ; \alpha, T)\right| d R \\
& \quad \leqq\left(\frac{1}{T} \int_{0}^{T}\left|F_{\alpha}(R ; T)-\sum_{m \leqq N} f_{m}(\sqrt{m} R ; \alpha, T)\right|^{2} d R\right)^{1 / 2} \leqq C_{0} N^{-1 / 6} .
\end{aligned}
$$

Hence by Lemmas $3.2,3.4$, when $T \geqq N^{2}$,

$$
\frac{1}{T} \int_{0}^{T}\left|F_{\alpha}(R)-\sum_{m \leqq N} f_{m}(\sqrt{m} R ; \alpha)\right| d R \leqq C_{1} N^{-1 / 6}
$$

from which Theorem 3.1 follows.

Proof of Lemma 3.2. If $|x-\alpha| \leqq R$, then

$$
\begin{aligned}
\left|\chi_{\alpha}(x ; R, T)-\chi_{\alpha}(x ; R)\right| & =\int_{|y-\alpha| \geqq R} \pi T^{2} \exp \left(-\pi^{2} T^{2}|x-y|^{2}\right) d y \\
& \leqq \int_{|x-y| \geqq R-|x-\alpha|} \pi T^{2} \exp \left(-\pi^{2} T^{2}|x-y|^{2}\right) d y \\
& =\exp \left(-\pi^{2} T^{2}(R-|x-\alpha|)^{2}\right) .
\end{aligned}
$$

Similarly, if $|x-\alpha| \geqq R$, then

$$
\begin{aligned}
\left|\chi_{\alpha}(x ; R, T)-\chi_{\alpha}(x ; R)\right| & =\chi_{\alpha}(x ; R, T)=\int_{|y-\alpha| \leqq R} \pi T^{2} \exp \left(-\pi^{2} T^{2}|x-y|^{2}\right) d y \\
& \leqq \int_{|x-y| \geqq|x-\alpha|-R} \pi T^{2} \exp \left(-\pi^{2} T^{2}|x-y|^{2}\right) d y \\
& =\exp \left(-\pi^{2} T^{2}(R-|x-\alpha|)^{2}\right) .
\end{aligned}
$$

Hence (3.4) holds for all $x$.

From (3.4),

$$
\frac{1}{T} \int_{0}^{T}\left|F_{\alpha}(R ; T)-F_{\alpha}(R)\right| d R \leqq \frac{1}{T} \int_{0}^{T} \sum_{n \in \mathbf{Z}^{2}} \exp \left(-\pi^{2} T^{2}(R-|n-\alpha|)^{2}\right) \frac{d R}{\sqrt{R}}
$$


For $|n-\alpha| \leqq T$

$$
\int_{0}^{T} \exp \left(-\pi^{2} T^{2}(R-|n-\alpha|)^{2}\right) \frac{d R}{\sqrt{R}} \leqq \frac{C}{T \sqrt{|n-\alpha|}},
$$

(since the main contribution to the integral comes from a small neighborhood of the point $R=|n-\alpha|)$. Hence

$$
\begin{aligned}
\sum_{|n-\alpha| \leqq T} \frac{1}{T} \int_{0}^{T} \exp \left(-\pi^{2} T^{2}(R-|n-\alpha|)^{2}\right) \frac{d R}{\sqrt{R}} \\
\leqq \frac{C}{T^{2}} \sum_{|n-\alpha| \leqq T} \frac{1}{\sqrt{|n-\alpha|}} \leqq C_{0} T^{-1 / 2}
\end{aligned}
$$

When $|n-\alpha| \geqq T$,

$$
\int_{0}^{T} \exp \left(-\pi^{2} T^{2}(R-|n-\alpha|)^{2}\right) \frac{d R}{\sqrt{R}} \leqq \frac{C}{\sqrt{T}} \exp \left(-\pi^{2} T^{2}(|n-\alpha|-T)^{2}\right),
$$

(since the main contribution to this integral comes from a small neighborhood of the point $R=T$ ), hence

$$
\begin{aligned}
\sum_{|n-\alpha| \geqq T} & \frac{1}{T} \int_{0}^{T} \exp \left(-\pi^{2} T^{2}(R-|n-\alpha|)^{2}\right) \frac{d R}{\sqrt{R}} \\
& \leqq \frac{C}{T^{3 / 2}} \sum_{|n-\alpha| \geqq T} \exp \left(-\pi^{2} T^{2}(|n-\alpha|-T)^{2}\right) \leqq C_{0} T^{-1 / 2}
\end{aligned}
$$

From (3.5)-(3.7) Lemma 3.2 follows.

Proof of Lemma 3.3. From the Poisson summation formula,

$$
\begin{aligned}
F_{\alpha}(R ; T) & =\sum_{n \neq 0} \tilde{\chi}_{\alpha}(2 \pi n ; R, T) / \sqrt{R} \\
& =\sum_{n \neq 0} \exp \left(-|n|^{2} / T^{2}\right) \tilde{\chi}_{\alpha}(2 \pi n ; R) / \sqrt{R}, \sum_{n \neq 0}=\sum_{n \in \mathbf{Z}^{2} \backslash\{0\}},
\end{aligned}
$$

hence (1.10) leads us to

$$
F_{\alpha}(R ; T)=\sum_{m=1}^{\infty} f_{m}(\sqrt{m} R ; \alpha, T)+O\left(R^{-1}\right) .
$$

Therefore Lemma 3.3 will follow if we prove that

$$
\frac{1}{T} \int_{0}^{T}\left|\sum_{m>N} f_{m}(\sqrt{m} R ; \alpha, T)\right|^{2} d R \leqq C\left(T^{-1 / 2}+N^{-1 / 3}\right) .
$$

Observe that

$$
\sum_{m>N} f_{m}(\sqrt{m} R ; \alpha, T)=\pi^{-1} \sum_{n \in S(N)} \frac{\exp \left(-|n|^{2} / T^{2}\right)}{|n|^{3 / 2}} \cos \left(2 \pi|n| R-\frac{3 \pi}{4}\right) e(n \alpha)
$$

where

$$
S(N)=\left\{\left.n \in \mathbf{Z}^{2}|| n\right|^{2}=m k^{2} \text { with } m>N\right\}
$$


If $n \in S(N)$ then clearly $|n|>\sqrt{N}$. From (3.9),

$$
\begin{aligned}
\frac{1}{T} \int_{0}^{T} \mid & \left.\sum_{m>N} f_{m}(\sqrt{m} R ; \alpha, T)\right|^{2} d R \\
= & \pi^{-2} \sum_{n, n^{\prime} \in S(N)} \frac{\exp \left(-\left(|n|^{2}+\left|n^{\prime}\right|^{2}\right) / T^{2}\right)}{|n|^{3 / 2}\left|n^{\prime}\right|^{3 / 2}} e\left(\left(n-n^{\prime}\right) \alpha\right) \\
& \times \frac{1}{T} \int_{0}^{T} \cos \left(2 \pi|n| R-\frac{3 \pi}{4}\right) \cos \left(2 \pi\left|n^{\prime}\right| R-\frac{3 \pi}{4}\right) d R .
\end{aligned}
$$

From the formula of product of cosines, we obtain

$$
\left|\int_{0}^{T} \cos \left(2 \pi|n| R-\frac{3 \pi}{4}\right) \cos \left(2 \pi\left|n^{\prime}\right| R-\frac{3 \pi}{4}\right) d R\right| \leqq \min \left\{T,|| n|-| n^{\prime}||^{-1}\right\},
$$

hence

$$
\begin{aligned}
\frac{1}{T} \int_{0}^{T} \mid & \left.\sum_{m>N} f_{m}(\sqrt{m} R ; \alpha, T)\right|^{2} d R \\
& \leqq \frac{1}{T} \sum_{n, n^{\prime} \in S(N)} \frac{\exp \left(-\left(|n|^{2}+\left|n^{\prime}\right|^{2}\right) / T^{2}\right)}{|n|^{3 / 2}\left|n^{\prime}\right|^{3 / 2}} \min \left\{T,|| n|-| n^{\prime}||^{-1}\right\} \\
& \leqq \frac{1}{T} \sum_{|n|,\left|n^{\prime}\right|>\sqrt{N}} \frac{\exp \left(-\left(|n|^{2}+\left|n^{\prime}\right|^{2}\right) / T^{2}\right)}{|n|^{3 / 2}\left|n^{\prime}\right|^{3 / 2}} \min \left\{T,|| n|-| n^{\prime}||^{-1}\right\}
\end{aligned}
$$

Straightforward computations give

$$
\begin{gathered}
\frac{1}{T} \sum_{|n|,\left|n^{\prime}\right|>\sqrt{N}} \frac{\exp \left(-\left(|n|^{2}+\left|n^{\prime}\right|^{2}\right) / T^{2}\right)}{|n|^{3 / 2}\left|n^{\prime}\right|^{3 / 2}} \min \left\{T,|| n|-| n^{\prime}||^{-1}\right\} \\
\leqq C\left(T^{-1 / 2}+N^{-1 / 3}\right) .
\end{gathered}
$$

Hence we have (3.8), and Lemma 3.3 follows.

Let us sketch the proof of (3.10). The sum over the set

$$
S_{0}(N)=\left\{n, n^{\prime}|| n|,| n^{\prime}|>\sqrt{N} ;||n|-\left|n^{\prime}\right| \mid>10\right\}
$$

is estimated by integral,

$$
\leqq C \int_{\sqrt{N}}^{\infty} \int_{\sqrt{N}}^{\infty} \frac{\exp \left(-\left(|x|^{2}+\left|x^{\prime}\right|^{2}\right) / T^{2}\right)}{|x|^{3 / 2}\left|x^{\prime}\right|^{3 / 2}\left(1+|| x|-| x^{\prime}||\right)} d x d x^{\prime} \leqq C_{0} \log ^{2} T .
$$

To estimate the sum over the complementary set

$$
S_{1} \cdot(N)=\left\{n, n^{\prime}|| n|,| n^{\prime}|>\sqrt{N} ;||n|-\left|n^{\prime}\right| \mid \leqq 10\right\}
$$

let us fix $n$ and define the annuli

$$
S_{1}(N, n ; k)=\left\{n^{\prime}\left|\frac{k}{4 n} \leqq\right| n^{\prime}|-| n \mid \leqq \frac{k+1}{4 n}\right\} \quad k=0,1, \ldots, 40|n| .
$$


If $n^{\prime}, n^{\prime \prime} \in S_{1}(N, n ; k)$, then

$$
\frac{1}{4|n|} \geqq|| n^{\prime}|-| n^{\prime \prime}||=\frac{\left.|| n^{\prime}\right|^{2}-\left|n^{\prime \prime}\right|^{2} \mid}{\left|n^{\prime}\right|+\left|n^{\prime \prime}\right|} \geqq \frac{\left.|| n^{\prime}\right|^{2}-\left|n^{\prime \prime}\right|^{2} \mid}{2(|n|+10)} .
$$

Without loss of generality we may assume that $\sqrt{N}>20$ (say), $|n|>20$, so $|n|+10<2|n|$, and the last relation implies

$$
\left.|| n^{\prime}\right|^{2}-\left|n^{\prime \prime}\right|^{2} \mid<1 \text {. }
$$

Hence $\left|n^{\prime}\right|^{2}=\left|n^{\prime \prime}\right|^{2}$, and the number of elements in $S_{1}(N, n ; k)$ does not exceed the number of representations of an integer $m=\left|n^{\prime}\right|^{2}$ as a sum of two squares, which is bounded by $C_{\varepsilon}|n|^{\varepsilon}$. Hence

$$
\begin{aligned}
& \sum_{S_{1}(N)} \frac{\exp \left(-\left(|n|^{2}+\left|n^{\prime}\right|^{2}\right) / T^{2}\right)}{|n|^{3 / 2}\left|n^{\prime}\right|^{3 / 2}} \min \left\{T,|| n|-| n^{\prime}||^{-1}\right\} \\
& \leqq C \sum_{|n| \geqq \sqrt{N}} \frac{\exp \left(-|n|^{2} / T^{2}\right)}{|n|^{3}}\left(T+\sum_{k=1}^{40|n|} \frac{|n|}{k}\right) C_{\varepsilon}|n|^{\varepsilon} \\
& \leqq C_{\varepsilon}^{\prime}\left(T N^{(1-\varepsilon) / 2}+T^{2 \varepsilon}\right) .
\end{aligned}
$$

(3.10) follows from (3.11), (3.12).

Proof of Lemma 3.4. Observe that

$$
\left|\sum_{n:|n|^{2}=m} e(n \alpha)\right| \leqq r(m) \leqq C_{\varepsilon} m^{\varepsilon}
$$

for every $\varepsilon>0$, where $r(m)$ is the number of representations of $m$ as a sum of two squares. So setting $\varepsilon=0.01$, we obtain from (1.13), (3.3) that, if $T \geqq m^{1 / 2}$ then

$$
\begin{aligned}
\left|f_{m}(t ; \alpha, T)-f_{m}(t ; \alpha)\right| & \leqq C \sum_{k=1}^{\infty} \frac{\left|1-\exp \left(-m k^{2} / T^{2}\right)\right|}{\left(m k^{2}\right)^{0.74}} \\
& =C\left(\sum_{k m k^{2} \leqq T^{2}} \ldots+\sum_{k \cdot m k^{2}>T^{2}} \ldots\right) \\
& \leqq C\left(\sum_{k \cdot m k^{2} \leqq T^{2}} \frac{m k^{2} / T^{2}}{\left(m k^{2}\right)^{0.74}}+\sum_{k: m k^{2}>T^{2}} \frac{1}{\left(m k^{2}\right)^{0.74}}\right) \\
& \leqq C_{1} m^{-1 / 2} T^{-0.48} .
\end{aligned}
$$

Indeed,

$$
\begin{aligned}
\sum_{k . m k^{2} \leqq T^{2}} \frac{m k^{2} / T^{2}}{\left(m k^{2}\right)^{0.74}} & =\sum_{k: m k^{2} \leqq T^{2}}\left(m k^{2}\right)^{0.26} / T^{2} \\
& \leqq C_{0}\left(\left(T^{2}\right)^{0.26} / T^{2}\right)\left(T / m^{1 / 2}\right)=C_{0} m^{-1 / 2} T^{-0.48}
\end{aligned}
$$

and

$$
\sum_{k: m k^{2}>T^{2}} \frac{1}{\left(m k^{2}\right)^{0.74}} \leqq C_{0} m^{-0.74}\left(T / m^{1 / 2}\right)^{-0.48}=C_{0} m^{-1 / 2} T^{-0.48}
$$


which was stated. We obtain now that, if $T \geqq N^{1 / 2}$, then

$$
\sum_{m \leqq N}\left|f_{m}(t ; \alpha, T)-f_{m}(t ; \alpha)\right| \leqq C_{2} N^{1 / 2} T^{-0.48},
$$

from which Lemma 3.4 follows.

Theorem 3.5. Hypothesis $\left(H_{0}\right)$ holds for $F_{\alpha}(R)$ with $a_{n}(t)=f_{m}(t ; \alpha)$, where $m=m(n)$ is the $n$-th square free integer.

Proof. The condition $\int_{0}^{1} f_{m}(t ; \alpha) d t=0$ is evident from (1.13), which implies (1.15). In addition, from (1.13),

$$
\left|f_{m}(t ; \alpha)\right| \leqq m^{-3 / 4} \sum_{k=1}^{\infty} k^{-3 / 2} r\left(m k^{2}\right)
$$

where $r(m)$ is the number of representations of $m$ as a sum of two squares. As is well known (see [HW]),

$$
r(m)<C_{\varepsilon} m^{\varepsilon}
$$

for every $\varepsilon>0$, hence

$$
\left|f_{m}(t ; \alpha)\right| \leqq m^{-(3 / 4)+\varepsilon} C_{\varepsilon} \sum_{k=1}^{\infty} k^{-(3 / 2)+\varepsilon}<C_{\varepsilon}^{\prime} m^{-(3 / 4)+\varepsilon} \leqq C_{\varepsilon}^{\prime} n^{-(3 / 4)+\varepsilon}
$$

This implies (1.16). The condition (1.17) follows from Theorem 3.1.

Theorem 3.6. For every probability density $\rho(x)$ on $[0,1]$ and for every continuous bounded function $g(x)$ on $\mathbf{R}^{1}$,

$$
\lim _{T \rightarrow \infty} \frac{1}{T} \int_{0}^{T} g\left(F_{\alpha}(R)\right) \rho(t / T) d t=\int_{-\infty}^{\infty} g(x) v_{\alpha}(d x),
$$

where $v_{\alpha}(d x)$ is the distribution of the random series

$$
\eta_{\alpha}=\sum_{m=1}^{\infty} f_{m}\left(t_{m} ; \alpha\right)
$$

and $t_{m}$ are independent random variables uniformly distributed on $[0,1]$.

Proof. It follows from Theorems 1.2, 3.5.

\section{Upper Bound on the Error Term Distribution Density}

In this section we study some properties of the distribution $v_{\alpha}(d x)$ defined in Theorem 3.6. These properties are needed to complete the proof of Theorem 1.1. Our study is based on the following general theorem:

Theorem 4.1. Assume that $a_{n}(t), n \geqq 1$, are real valued continuous functions, periodic with period 1 , satisfying the conditions:

(i) $\int_{0}^{1} a_{n}(t) d t=0$;

(ii) $\sup _{0 \leqq t \leqq 1}\left|a_{n}(t)\right|<J n^{-\gamma}$ with some $J>0$ and $\frac{1}{2}<\gamma<1$;

(iii) $\sum_{j=n}^{\infty} \int_{0}^{1} a_{j}(t)^{2} d t>J_{0} n^{-\lambda}$ with some $J_{0}>0$ and $2 \gamma-1 \leqq \lambda<\gamma$. 
Then the distribution of the random series $\eta=\sum_{n=1}^{\infty} a_{n}\left(t_{n}\right)$, where $t_{1}, t_{2}, \ldots$ are independent random variables uniformly distributed on $[0,1]$, is absolutely continuous with respect to Lebesgue measure, and the distribution density $p(x)$ of $\eta$ can be extended to the whole complex plane $\mathbf{C}$ as an entire function of $x$. For real $x$,

$$
0 \leqq p(x) \leqq C_{0} \exp \left(-C|x|^{(2 \gamma-\lambda) /(1-\gamma)}\right)
$$

with $C, C_{0}>0$.

Proof of Theorem 4.1 is given in Appendix A.

Theorem 4.2. For every $\alpha \in \mathbf{R}^{2}$, the functions $a_{n}(t)=f_{n}(t ; \alpha)$ satisfy the conditions (i)-(iii) of Theorem 4.1 with $\gamma=(3 / 4)-\varepsilon$ and $\lambda=(1 / 2)+\varepsilon$ for every $\varepsilon>0$.

Corollary. The distribution $v_{\alpha}(d x)$ is absolutely continuous with respect to Lebesgue measure, $v_{\alpha}(d x)=p_{\alpha}(x) d x$. The density $p_{\alpha}(x)$ can be extended to an entire function and satisfies (1.3) for real $x$.

Proof of Corollary. It follows from Theorems 4.1, 4.2.

Proof of Theorem 4.2. From (1.13),

$$
f_{m}(t ; \alpha)=\pi^{-1} \sum_{k=1}^{\infty} \frac{r_{\alpha}\left(m k^{2}\right)}{\left(m k^{2}\right)^{3 / 4}} \cos \left(2 \pi k t-\frac{3 \pi}{4}\right),
$$

where $r_{\alpha}(m)$ was defined before,

$$
r_{\alpha}(m)=\sum_{n_{1}^{2}+n_{2}^{2}=m} e\left(\alpha_{1} n_{1}+\alpha_{2} n_{2}\right) .
$$

The condition (i) is evident from (4.1). For the condition (ii),

$$
\sup _{0 \leqq t \leqq 1}\left|f_{m}(t ; \alpha)\right|<J_{\varepsilon} m^{-3 / 4+\varepsilon},
$$

see (3.16). The main problem is to verify (iii),

$$
\sum_{m \geqq N} \int_{0}^{1} f_{m}(t ; \alpha)^{2} d t \geqq C_{\varepsilon, \alpha} N^{-1 / 2-\varepsilon} .
$$

From (4.1) we have:

$$
\int_{0}^{1} f_{m}(t ; \alpha)^{2} d t=\frac{\pi^{-2}}{2} \sum_{k=1}^{\infty} \frac{\left|r_{\alpha}\left(m k^{2}\right)\right|^{2}}{\left(m k^{2}\right)^{3 / 2}} .
$$

We will deduce (4.3) from the estimate

$$
\sum_{n \geqq N} \frac{\left|r_{\alpha}(n)\right|^{2}}{n^{3 / 2}} \geqq C_{\varepsilon, \alpha}^{\prime} N^{-1 / 2-\varepsilon} .
$$

The proof of (4.5) is based on the following theorem:

Theorem 4.3. For every $\alpha \in \mathbf{R}^{2}$,

$$
\lim _{b \rightarrow \infty} \inf (1 / b) \sum_{n=1}^{\infty}\left|r_{\alpha}(n)\right|^{2} \exp (-n / b) \geqq \pi .
$$


Corollary. For every $\alpha \in \mathbf{R}^{2}$,

$$
\inf _{b \geqq 1}(1 / b) \sum_{n=1}^{\infty}\left|r_{\alpha}(n)\right|^{2} \exp (-n / b)>0 .
$$

The proof of Theorem 4.3 will be given in Appendix B. Let us show that (4.6) implies

$$
\sum_{n=1}^{N}\left|r_{\alpha}(n)\right|^{2} \geqq \frac{N}{\log N}
$$

when $N \geqq N_{0}(\alpha)$. Indeed,

$$
\sum_{n=1}^{N}\left|r_{\alpha}(n)\right|^{2} \geqq \sum_{n=1}^{N}\left|r_{\alpha}(n)\right|^{2} \exp (-n / b) .
$$

Let us fix some $\varepsilon>0$ and put $b=N /(2 \varepsilon \log N), \varepsilon>0$. Then by (4.6)

$$
\sum_{n=1}^{\infty}\left|r_{\alpha}(n)\right|^{2} \exp (-n / b) \geqq \varepsilon_{0} b=\varepsilon_{0} \frac{N}{2 \varepsilon \log N}
$$

with some $\varepsilon_{0}=\varepsilon_{0}(\alpha)>0$, which does not depend on $b$ and hence on $N$ and $\varepsilon$. On the other hand, $\left|r_{\alpha}(n)\right|^{2} \leqq C_{\varepsilon} n^{\varepsilon}$, so

$$
\begin{aligned}
\sum_{n>N}\left|r_{\alpha}(n)\right|^{2} \exp (-n / b) & \leqq C_{\varepsilon} \sum_{n>N} n^{\varepsilon} \exp (-n / b) \leqq C_{\varepsilon}^{\prime} N^{\varepsilon} b \exp (-N / b) \\
& =C_{\varepsilon}^{\prime} \frac{N^{1+\varepsilon}}{2 \varepsilon \log N} \exp \left(-\log N^{2 \varepsilon}\right)=C_{\varepsilon}^{\prime} \frac{N^{1-\varepsilon}}{2 \varepsilon \log N} \leqq \frac{N}{\log N}
\end{aligned}
$$

when $N \geqq N_{1}(\varepsilon)$. Hence

$$
\sum_{n=1}^{N}\left|r_{\alpha}(n)\right|^{2} \exp (-n / b) \geqq \varepsilon_{0} \frac{N}{2 \varepsilon \log N}-\frac{N}{\log N}=\frac{N}{\log N}
$$

when $\varepsilon=\varepsilon_{0} / 4$ and $N \geqq N_{1}\left(\varepsilon_{0} / 4\right), \varepsilon_{0}=\varepsilon_{0}(\alpha)$. Thus (4.7) is proved.

Let us prove (4.5). For all $\delta>0$,

$$
\sum_{n \geqq N} \frac{\left|r_{\alpha}(n)\right|^{2}}{n^{3 / 2}} \geqq \sum_{n=N}^{N^{1+\delta}} \frac{\left|r_{\alpha}(n)\right|^{2}}{n^{3 / 2}} \geqq N^{-(3 / 2)(1+\delta)} \sum_{n=N}^{N^{1+\delta}}\left|r_{\alpha}(n)\right|^{2}
$$

By (4.7)

$$
\sum_{n=1}^{N^{1+\delta}}\left|r_{\alpha}(n)\right|^{2} \geqq \frac{N^{1+\delta}}{(1+\delta) \log N} \geqq N^{1+\delta / 2}
$$

when $N \geqq N_{0}(\alpha, \delta)$. On the other hand,

$$
\sum_{n=1}^{N-1}\left|r_{\alpha}(n)\right|^{2} \leqq C_{\delta} \sum_{n=1}^{N-1} n^{\delta / 4} \leqq \frac{N^{1+\delta / 2}}{2}
$$

when $N \geqq N_{1}(\delta)$, so

$$
\sum_{n=N}^{N^{1+\delta}}\left|r_{\alpha}(n)\right|^{2} \geqq N^{1+\delta / 2}-\frac{N^{1+\delta / 2}}{2}=\frac{N^{1+\delta / 2}}{2}
$$


and

$$
\sum_{n \geqq N} \frac{\left|r_{\alpha}(n)\right|^{2}}{n^{3 / 2}} \geqq \sum_{n=N}^{N^{1+\delta}} \frac{\left|r_{\alpha}(n)\right|^{2}}{n^{3 / 2}} \geqq N^{-(3 / 2)(1+\delta)} \frac{1}{2} N^{1+\delta / 2}=\frac{1}{2} N^{-1 / 2-\delta}
$$

when $N \geqq \max \left\{N_{0}(\alpha, \delta), N_{1}(\delta)\right\}$. Thus (4.5) is proved.

Let us turn now to the proof of (4.3). We have for all $\delta>0$ :

$$
\begin{aligned}
\sum_{m \geqq N} \int_{0}^{1} f_{m}(t ; \alpha)^{2} d t & =\frac{\pi^{-2}}{2} \sum_{m \geqq N} \sum_{k=1}^{\infty} \frac{\left|r_{\alpha}\left(m k^{2}\right)\right|^{2}}{\left(m k^{2}\right)^{3 / 2}} \\
& \geqq \frac{\pi^{-2}}{2} \sum_{m \geqq N} \sum_{\left\{k \mid m k^{2} \geqq N^{1+\delta}\right\}} \frac{\left|r_{\alpha}\left(m k^{2}\right)\right|^{2}}{\left(m k^{2}\right)^{3 / 2}} .
\end{aligned}
$$

The last double sum is equal to

$$
\begin{aligned}
\sum_{\left\{n: n \geqq N^{1+\delta} ; n=m k^{2}, m \in Q, m \geqq N\right\}} \frac{\left|r_{\alpha}(n)\right|^{2}}{n^{3 / 2}}= & \sum_{n \geqq N^{1+\delta}} \frac{\left|r_{\alpha}(n)\right|^{2}}{n^{3 / 2}} \\
& -\sum_{\left\{n: n \geqq N^{1+\delta} ; n=m k^{2}, m \in Q, m<N\right\}} \frac{\left|r_{\alpha}(n)\right|^{2}}{n^{3 / 2}},
\end{aligned}
$$

where $Q$ is the set of square-free natural numbers. By (4.5)

$$
\sum_{n \geqq N^{1+\delta}} \frac{\left|r_{\alpha}(n)\right|^{2}}{n^{3 / 2}} \geqq C_{\varepsilon, \alpha} N^{-(1 / 2+\varepsilon)(1+\delta)} .
$$

On the other hand, if $m<N$ and $m k^{2} \geqq N^{1+\delta}$, then $k>N^{\delta / 2}$, so for $m<N$,

$$
\begin{aligned}
\sum_{\left\{k: m k^{2} \geqq N^{1+\delta}\right\}} \frac{\left|r_{\alpha}\left(m k^{2}\right)\right|^{2}}{\left(m k^{2}\right)^{3 / 2}} & \leqq \sum_{k>N^{\delta / 2}} \frac{\left|r_{\alpha}\left(m k^{2}\right)\right|^{2}}{\left(m k^{2}\right)^{3 / 2}} \leqq \sum_{k>N^{\delta / 2}} \frac{r\left(m k^{2}\right)^{2}}{\left(m k^{2}\right)^{3 / 2}} \\
& \leqq C_{\varepsilon} \sum_{k>N^{\delta / 2}}\left(m k^{2}\right)^{-3 / 2+\varepsilon} \leqq C_{\varepsilon}^{\prime} m^{-3 / 2+\varepsilon} N^{-\delta(1-\varepsilon)}
\end{aligned}
$$

Therefore

$$
\sum_{\left\{n: n \geqq N^{1+\delta} ; n=m k^{2}, m \in Q, m<N\right\}} \frac{\left|r_{\alpha}(n)\right|^{2}}{n^{3 / 2}} \leqq C_{\varepsilon}^{\prime} N^{-\delta(1-\varepsilon)} \sum_{m<N} m^{-3 / 2+\varepsilon} \leqq C_{\varepsilon}^{\prime \prime} N^{-1 / 2-\delta+\varepsilon+\delta \varepsilon} .
$$

Combining (4.9)-(4.11) with (4.8),

$$
\begin{aligned}
\sum_{m \geqq N} \int_{0}^{1} f_{m}(t ; \alpha)^{2} d t & \geqq C_{\varepsilon, \alpha} N^{-(1 / 2+\varepsilon)(1+\delta)}-C_{\varepsilon}^{\prime \prime} N^{-1 / 2-\delta+\varepsilon+\delta \varepsilon} \\
& =N^{-(1 / 2+\varepsilon)(1+\delta)}\left(C_{\varepsilon, \alpha}-C_{\varepsilon}^{\prime \prime} N^{-\delta / 2+2 \varepsilon+2 \delta \varepsilon}\right) .
\end{aligned}
$$

Choosing now $\delta=8 \varepsilon$, we get for small $\varepsilon>0$,

$$
-\frac{\delta}{2}+2 \varepsilon+2 \delta \varepsilon=-2 \varepsilon+16 \varepsilon^{2}<-\varepsilon
$$

so for $N \geqq N_{0}(\varepsilon, \alpha)$

$$
\sum_{m \geqq N} \int_{0}^{1} f_{m}(t ; \alpha)^{2} d t \geqq C_{\varepsilon, \alpha}^{\prime} N^{-(1 / 2+\varepsilon)(1+8 \varepsilon)},
$$

which proves (4.3). Theorem 4.2 is thus proved. 


\section{Lower Bound on the Error Term Distribution Function}

In this section we prove the following theorem:

Theorem 5.1. For every $\varepsilon>0, x_{0}=x_{0}(\varepsilon)>0$ exists such that

$$
\begin{gathered}
\operatorname{Pr}\left\{\sum_{m=1}^{\infty} f_{m}\left(t_{m} ; \alpha\right)>x\right\}>\exp \left(-x^{4+\varepsilon}\right), \\
\operatorname{Pr}\left\{\sum_{m=1}^{\infty} f_{m}\left(t_{m} ; \alpha\right)<-x\right\}>\exp \left(-x^{4+\varepsilon}\right),
\end{gathered}
$$

when $x>x_{0}$.

Proof. We prove the first inequality. The second one can be proved in the same way.

We start with several definitions. Let $\varepsilon>0$ be an arbitrary small number. We will distinguish for $m \leqq n$ between "good" $m$ 's, "bad" $m$ 's and "neutral" $m$ 's. Define the set of "good" $m$ 's as

$$
G_{n}=\left\{1 \leqq m \leqq n|| r_{\alpha}(m) \mid \geqq m^{-\varepsilon}\right\},
$$

and $G_{n}^{c}=Z_{n} \backslash G_{n}$ with $Z_{n}=\{1,2, \ldots, n\}$. By $|G|$ we denote the number of elements in a finite set $G$.

Lemma 5.2. For every $\varepsilon>0, n_{0}=n_{0}(\varepsilon)$ exists such that $\left|G_{n}\right|>n^{1-3 \varepsilon}$ when $n>n_{0}(\varepsilon)$.

Proof. We have:

$$
\sum_{m \in G_{n}^{c}}\left|r_{\alpha}(m)\right|^{2} \leqq \sum_{m=1}^{n} m^{-2 \varepsilon}<2 n^{1-2 \varepsilon}
$$

so by (4.7),

$$
\sum_{m \in G_{n}}\left|r_{\alpha}(m)\right|^{2} \geqq \frac{n}{\log n}-2 n^{1-2 \varepsilon}>n^{1-\varepsilon}
$$

when $n>n_{0}(\varepsilon)$. On the other hand, $\left|r_{\alpha}(m)\right|^{2}<C_{\varepsilon} m^{\varepsilon} \leqq C_{\varepsilon} n^{\varepsilon}$, hence

Hence

$$
\sum_{m \in G_{n}}\left|r_{\alpha}(m)\right|^{2}<C_{\varepsilon} n^{\varepsilon}\left|G_{n}\right|
$$

$$
\left|G_{n}\right|>\left(C_{\varepsilon}\right)^{-1} n^{1-2 \varepsilon}>n^{1-3 \varepsilon},
$$

when $n>n_{1}(\varepsilon)$, which proves Lemma 5.2.

When $m \in G_{n}$, define

$$
A_{m}=\left\{t \mid f_{m}(t ; \alpha)>m^{-(3 / 4)-4 \varepsilon}\right\} .
$$

Lemma 5.3. For every $\varepsilon>0, n_{0}=n_{0}(\varepsilon)>0$ exists such that mes $A_{m}>m^{-10}$ for all $m \in G_{n}$ with $m, n>n_{0}$.

Proof. When $m \in G_{n}$,

$$
\int_{0}^{1}\left|f_{m}(t ; \alpha)\right|^{2} d t>C m^{-3 / 2}\left|r_{\alpha}(m)\right|^{2}>C m^{-(3 / 2)-2 \varepsilon}
$$


and by (3.16),

Hence

$$
\sup _{0 \leqq t \leqq 1}\left|f_{m}(t ; \alpha)\right|<C_{\varepsilon}^{\prime} m^{-(3 / 4)+\varepsilon}
$$

and

$$
\begin{aligned}
C_{\varepsilon}^{\prime} m^{-(3 / 4)+\varepsilon} \int_{0}^{1}\left|f_{m}(t ; \alpha)\right| d t & >\sup _{0 \leqq t \leqq 1}\left|f_{m}(t ; \alpha)\right| \int_{0}^{1}\left|f_{m}(t ; \alpha)\right| d t \\
& \geqq \int_{0}^{1}\left|f_{m}(t ; \alpha)\right|^{2} d t>C m^{-(3 / 2)-2 \varepsilon}
\end{aligned}
$$

$$
\int_{0}^{1}\left|f_{m}(t ; \alpha)\right| d t>C_{\varepsilon}^{\prime \prime} m^{-(3 / 4)-3 \varepsilon} \text {. }
$$

Define $f_{m}^{+}(t ; \alpha)=\max \left(0, f_{m}(t ; \alpha)\right)$ and $f_{m}^{-}(t ; \alpha)=\max \left(0,-f_{m}(t ; \alpha)\right)$. Since $\int_{0}^{1} f_{m}(t ; \alpha) d t=0,(5.2)$ implies that

$$
\int_{0}^{1} f_{m}^{+}(t ; \alpha) d t>C_{\varepsilon} m^{-(3 / 4)-3 \varepsilon}
$$

with $C_{\varepsilon}=(1 / 2) C_{\varepsilon}^{\prime \prime}$. It follows from the definition of $A_{m}$ and (5.1), that

so

$$
\int_{0}^{1} f_{m}^{+}(t ; \alpha) d t<\left(C_{\varepsilon}^{\prime} m^{-(3 / 4)+\varepsilon}\right) \operatorname{mes} A_{m}+m^{-(3 / 4)-4 \varepsilon},
$$

$$
\text { mes } \begin{aligned}
A_{m} & >\left(C_{\varepsilon}^{\prime} m^{-(3 / 4)+\varepsilon}\right)^{-1}\left(C_{\varepsilon} m^{-(3 / 4)-3 \varepsilon}-m^{-(3 / 4)-4 \varepsilon}\right)=\left(C_{\varepsilon}^{\prime}\right)^{-1}\left(C_{\varepsilon} m^{-4 \varepsilon}-m^{-5 \varepsilon}\right) \\
& >m^{-10}
\end{aligned}
$$

for $m>n_{0}(\varepsilon)$. Lemma 5.3 is proved.

Define the set of "bad" $m$ 's as

$$
B_{n}=\left\{1 \leqq m \leqq n \mid \operatorname{mes}\left\{t: f_{m}(t ; \alpha)>0\right\}<m^{-10}\right\} .
$$

When $m \in B_{n}$, define

$$
A_{m}=\left\{t \mid f_{m}(t ; \alpha)>-m^{-5}\right\} .
$$

Lemma 5.4. When $m \in B_{n}$, mes $A_{m}>m^{-10}$.

Proof. When $m \in B_{n}$,

hence

$$
\int_{0}^{1} f_{m}^{+}(t ; \alpha) d t<m^{-10}
$$

and

$$
\int_{0}^{1} f_{m}^{-}(t ; \alpha) d t<m^{-10}
$$

$$
m^{-5} \operatorname{mes} A_{m}^{c}<m^{-10}, \quad A_{m}^{c}=[0,1] \backslash A_{m} .
$$

Hence mes $A_{m}^{c}<m^{-5}$, so mes $A_{m}>m^{-10}$.

Define the set of "neutral" $m$ 's as $N_{n}=Z_{n} \backslash\left(G_{n} \cup B_{n}\right)$. When $m \in N_{n}$, define

$$
A_{m}=\left\{t \mid f_{m}(t ; \alpha)>0\right\} \text {. }
$$

Since then $m \notin B_{n}$, mes $A_{m} \geqq m^{-10}$. 
Proof of Theorem 5.1. We have:

$$
\operatorname{Var} \sum_{m=n}^{\infty} f_{m}\left(t_{m} ; \alpha\right)=\sum_{m=n}^{\infty} \int_{0}^{1} f_{m}(t ; \alpha)^{2} d t<1
$$

when $n>n_{0}$. Hence by the Tchebyshev inequality

Define

$$
\operatorname{Pr}\left\{\left|\sum_{m=n}^{\infty} f_{m}\left(t_{m} ; \alpha\right)\right| \leqq 2\right\} \geqq 1-(1 / 4)=3 / 4 \text {. }
$$

$$
D_{n}=\left\{\left(t_{1}, t_{2}, \ldots\right)\left|t_{m} \in A_{m}, m=1,2, \ldots, n ;\right| \sum_{m=n+1}^{\infty} f_{m}\left(t_{m} ; \alpha\right) \mid \leqq 2\right\} .
$$

When $\left(t_{1}, t_{2}, \ldots\right) \in D_{n}$,

$$
\begin{aligned}
\sum_{m=1}^{\infty} f_{m}\left(t_{m} ; \alpha\right) & =\left(\sum_{G_{n}}+\sum_{B_{n}}+\sum_{N_{n}}+\sum_{m=n+1}^{\infty}\right) f_{m}\left(t_{m} ; \alpha\right) \\
& >n^{1-3 \varepsilon} n^{-(3 / 4)-4 \varepsilon}-\sum_{m=1}^{n} n^{-5}-2>n^{(1 / 4)-8 \varepsilon}
\end{aligned}
$$

$n>n_{0}(\varepsilon)$. On the other hand,

$$
\begin{aligned}
\operatorname{Pr} D_{n} & =\prod_{m=1}^{n} \operatorname{Pr} A_{m} \operatorname{Pr}\left\{\left|\sum_{m=n}^{\infty} f_{m}\left(t_{m} ; \alpha\right)\right| \leqq 2\right\}>C n^{-10 n}(3 / 4) \\
& =(3 / 4) C \exp (-10 n \log n)>\exp \left(-n^{1+\varepsilon}\right)
\end{aligned}
$$

when $n>n_{0}(\varepsilon)$. Setting $x=n^{(1 / 4)-8 \varepsilon}$, we obtain:

$$
\operatorname{Pr}\left\{\sum_{m=1}^{\infty} f_{m}\left(t_{m} ; \alpha\right)>x\right\}>\operatorname{Pr} D_{n}>\exp \left(-x^{(1+\varepsilon) /(1 / 4)-8 \varepsilon)}\right),
$$

$n>n_{0}(\varepsilon)$. Since $\varepsilon>0$ is arbitrarily small, Theorem 5.1 is proved.

Conclusion of Proof of Theorem 1.1. From Theorem 3.6 and Corollary of Theorem 4.2 it follows that (1.2) holds for every continuous bounded function $g(x)$. By implication, it holds for every piecewise continuous bounded function. Properties of $p_{\alpha}(x)$ follow from Corollary of Theorem 4.2 and Theorem 5.1.

\section{Appendix A. Proof of Theorem 4.1}

Let

$$
\chi_{j}(z)=\int_{0}^{1} \exp \left(i z a_{j}(t)\right) d t
$$

be the characteristic function of $a_{j}(t)$. Then the characteristic function of $\eta=\sum_{j=1}^{\infty} a_{j}\left(t_{j}\right)$ is

$$
\chi(z)=\prod_{j=1}^{\infty} \chi_{j}(z)
$$

Lemma A.1. $\chi(z)$ is an entire function of $z=\sigma+i \tau$ and

$$
|\chi(z)| \leqq \exp \left(-A \sigma^{2}\left(1+|z|^{1 / \gamma}\right)^{-\lambda}+B|\tau||z|^{(1-\gamma) / \gamma}\right),
$$

with some $A, B>0$ depending only on the constants $\gamma, J, J_{0}$ in the conditions (ii), (iii). 
Proof of Lemma A.1. Let us fix some $z \in \mathbf{C}$ and define

$$
n=n(z)=\left[\varepsilon^{-1}|z|^{1 / \gamma}\right]+1
$$

where $0<\varepsilon<1$ will be chosen later. For $j \leqq n-1$ we estimate $\left|\chi_{j}(z)\right|$ as

$$
\left|\chi_{j}(z)\right|=\left|\int_{0}^{1} \exp \left(i z a_{j}(t)\right) d t\right| \leqq \exp \left(|\tau|\left\|a_{j}(t)\right\|\right),
$$

where $\left\|a_{j}(t)\right\|=\sup _{0} \leqq t \leqq 1\left|a_{j}(t)\right|$. So

$$
\prod_{j \leqq n-1}\left|\chi_{j}(z)\right| \leqq \exp \left(|\tau| \sum_{j \leqq n-1}\left\|a_{j}(t)\right\|\right) .
$$

The condition (ii) implies that

$$
\sum_{j \leqq n-1}\left\|a_{j}(t)\right\| \leqq C(n-1)^{1-\gamma} .
$$

Due to (A.1), $(n-1) \leqq \varepsilon^{-1}|z|^{1 / \gamma}$, so

Hence

$$
\sum_{j \leqq n-1}\left\|a_{j}(t)\right\| \leqq C \varepsilon^{\gamma-1}|z|^{(1-\gamma) / \gamma} .
$$

$$
\prod_{j \leqq n-1}\left|\chi_{j}(z)\right| \leqq \exp \left(C \varepsilon^{\gamma-1}|\tau||z|^{(1-\gamma) / \gamma}\right) .
$$

Let us estimate now $\left|\chi_{j}(z)\right|$ for $j \geqq n$. By the Taylor formula

$$
\chi_{j}(z)=1-\frac{z^{2}}{2} \int_{0}^{1} a_{j}(t)^{2} d t+r_{j}(z)
$$

where

$$
\left|r_{j}(z)\right| \leqq \max _{|\zeta| \leqq|z|} \frac{1}{6}\left|\chi_{j}^{\prime \prime \prime}(\zeta)\right||z|^{3} \leqq \frac{|z|^{3}}{6} \int_{0}^{1}\left|a_{j}(t)\right|^{3} \exp \left(|z|\left|a_{j}(t)\right|\right) d t .
$$

Due to the condition (ii),

so

$$
|z|\left|a_{j}(t)\right| \leqq|z| J n^{-\gamma} \leqq J|z| \varepsilon^{\gamma}|z|^{-1}=J \varepsilon^{\gamma},
$$

$$
|z|\left|a_{j}(t)\right| \exp \left(|z|\left|a_{j}(t)\right|\right) \leqq J \varepsilon^{\gamma} \exp \left(J \varepsilon^{\gamma}\right) .
$$

Let us assume that $\varepsilon=\varepsilon(J)$ is so small that

$$
J \varepsilon^{\gamma} \exp \left(J \varepsilon^{\gamma}\right) \leqq \varepsilon^{1 / 4},
$$

(recall that $\gamma>1 / 2$ ). Then we get from (A.4), (A.5) that

$$
\left|r_{j}(z)\right| \leqq \varepsilon^{1 / 4}|z|^{2} \int_{0}^{1} a_{j}(t)^{2} d t .
$$

Let us write now $\chi_{j}(z)$ in the form

$$
\chi_{j}(z)=1-\frac{z^{2}}{2} \int_{0}^{1} a_{j}(t)^{2} d t+r_{j}(z)=\exp \left(-\frac{z^{2}}{2} \int_{0}^{1} a_{j}(t)^{2} d t+\delta_{j}(z)\right) .
$$


To estimate $\left|\delta_{j}(z)\right|$ we notice that by (A.6)

$$
|z|^{2}\left|a_{j}(t)\right|^{2} \leqq\left(J \varepsilon^{\gamma}\right)^{2} \leqq \varepsilon^{1 / 2}
$$

Since $|\ln (1+\delta)-\delta| \leqq \delta^{2}$, when $|\delta|<1 / 2$, we get from (A.7), (A.8),

$$
\left|\delta_{j}(z)\right| \leqq 2 \varepsilon^{1 / 4}|z|^{2} \int_{0}^{1} a_{j}(t)^{2} d t .
$$

Hence

$$
\begin{aligned}
\left|\chi_{j}(z)\right| & \leqq\left|\exp \left(-\frac{z^{2}}{2} \int_{0}^{1} a_{j}(t)^{2} d t\right)\right| \exp \left(\left|\delta_{j}(z)\right|\right) \\
& \leqq \exp \left(-\frac{\sigma^{2}-\tau^{2}}{2} \int_{0}^{1} a_{j}(t)^{2} d t+2 \varepsilon^{1 / 4}\left(\sigma^{2}+\tau^{2}\right) \int_{0}^{1} a_{j}(t)^{2} d t\right) \\
& =\exp \left(-\left(A_{0} \sigma^{2}-B_{0} \tau^{2}\right) \int_{0}^{1} a_{j}(t)^{2} d t\right),
\end{aligned}
$$

with $A_{0}=(1 / 2)-2 \varepsilon^{1 / 4}, B_{0}=(1 / 2)+2 \varepsilon^{1 / 4}$. Assuming that

we get

$$
2 \varepsilon^{1 / 4} \leqq 0.1
$$

Hence

$$
\left|\chi_{j}(z)\right| \leqq \exp \left(-\left(0.4 \sigma^{2}-0.6 \tau^{2}\right) \int_{0}^{1} a_{j}(t)^{2} d t\right)
$$

By (ii),

$$
\prod_{j \geqq n}\left|\chi_{j}(z)\right| \leqq \exp \left(-\left(0.4 \sigma^{2}-0.6 \tau^{2}\right) \sum_{j \geqq n} \int_{0}^{1} a_{j}(t)^{2} d t\right) .
$$

Due to (A.1),

$$
\begin{gathered}
\sum_{j \geqq n} \int_{0}^{1} a_{j}(t)^{2} d t \leqq J \sum_{j \geqq n} j^{-2 \gamma} \leqq C n^{1-2 \gamma} . \\
n^{1-2 \gamma} \leqq\left(\varepsilon^{-1}|z|^{1 / \gamma}\right)^{1-2 \gamma}=\varepsilon^{2 \gamma-1}|z|^{(1-2 \gamma) / \gamma},
\end{gathered}
$$

so

$$
\sum_{j \geqq n} \int_{0}^{1} a_{j}(t)^{2} d t \leqq C n^{1-2 \gamma} \leqq C \varepsilon^{2 \gamma-1}|z|^{(1-2 \gamma) / \gamma} \leqq C_{0}|\tau|^{(1-2 \gamma) / \gamma} .
$$

On the other hand, (iii) implies

so

$$
\sum_{j \geqq n} \int_{0}^{1} a_{j}(t)^{2} d t \geqq J_{0} n^{-\lambda} \geqq J_{0}\left(1+\varepsilon^{-1}|z|^{1 / \gamma}\right)^{-\lambda} \geqq C_{1}\left(1+|z|^{1 / \gamma}\right)^{-\lambda},
$$

$$
\prod_{j \geqq n}\left|\chi_{j}(z)\right| \leqq \exp \left(-0.4 C_{1} \sigma^{2}\left(1+|z|^{1 / \gamma}\right)^{-\lambda}+0.6 C_{0}|\tau|^{1 / \gamma}\right) .
$$

Setting $A=0.4 C_{1}, C=0.6 C_{0}$, we get

$$
\prod_{j \geqq n}\left|\chi_{j}(z)\right| \leqq \exp \left(-A \sigma^{2}\left(1+|\sigma+i \tau|^{1 / \gamma}\right)^{-\lambda}+C|\tau|^{1 / \gamma}\right) .
$$

This estimate together with (A.2) implies

$$
|\chi(z)|=\prod_{j=1}^{\infty}\left|\chi_{j}(z)\right| \leqq \exp \left\{-A \sigma^{2}\left(1+|z|^{1 / \gamma}\right)^{-\lambda}+C|\tau|^{1 / \gamma}+C^{\prime}|\tau||z|^{(1-\gamma) / \gamma}\right\} .
$$


Since $|\tau||z|^{(1-\gamma) / \gamma} \geqq|\tau|^{1 / \gamma}$, we get

$$
|\chi(z)| \leqq \exp \left\{-A \sigma^{2}\left(1+|z|^{1 / \gamma}\right)^{-\lambda}+B|\tau||z|^{(1-\gamma) / \gamma}\right\}
$$

with $B=C+C^{\prime}$. Lemma A.1 is proved.

From Lemma A.1 it follows that for real $\sigma$,

Since $\gamma>\lambda$,

$$
|\chi(\sigma)| \leqq \exp \left\{-A \sigma^{2}\left(1+|\sigma|^{1 / \gamma}\right)^{-\lambda}\right\}
$$

$$
p(x)=(2 \pi)^{-1} \int_{-\infty}^{\infty} \exp (-i x \sigma) \chi(\sigma) d \sigma
$$

is an entire function of $x$.

Let us estimate $p(x)$ for large real $x$. By the Cauchy formula

$$
\begin{aligned}
p(x) & =(2 \pi)^{-1} \int_{-\infty}^{\infty} \exp (-i x(\sigma-i \tau)) \chi(\sigma-i \tau) d \sigma \\
& =(2 \pi)^{-1} \exp (-x \tau) \int_{-\infty}^{\infty} \exp (-i x \sigma) \chi(\sigma-i \tau) d \sigma
\end{aligned}
$$

so

$$
p(x) \leqq(2 \pi)^{-1} \exp (-x \tau) \int_{-\infty}^{\infty}|\chi(\sigma-i \tau)| d \sigma
$$

From Lemma A.1,

$$
\int_{-\infty}^{\infty}|\chi(\sigma-i \tau)| d \sigma \leqq \int_{-\infty}^{\infty} \exp \left\{-A \sigma^{2}\left(1+|\sigma-i \tau|^{1 / \gamma}\right)^{-\lambda}+B|\tau||\sigma-i \tau|^{(1-\gamma) / \gamma}\right\} d \sigma .
$$

We will assume that $|\tau| \geqq 1$, so that $|\sigma-i \tau| \geqq 1$,

$$
\left(1+|\sigma-i \tau|^{1 / \gamma}\right)^{-\lambda} \geqq\left(2|\sigma-i \tau|^{1 / \gamma}\right)^{-\lambda} \geqq \frac{1}{2}|\sigma-i \tau|^{-\lambda / \gamma},
$$

and

$$
\int_{-\infty}^{\infty}|\chi(\sigma-i \tau)| d \sigma \leqq \int_{-\infty}^{\infty} \exp \left\{-\frac{A \sigma^{2}}{2}|\sigma-i \tau|^{-\lambda / \gamma}+B|\tau||\sigma-i \tau|^{(1-\gamma) / \gamma}\right\} d \sigma .
$$

Let us decompose the last integral into two integrals, over the regions $\{|\sigma| \leqq|\tau|\}$ and $\{|\sigma| \geqq|\tau|\}$. If $|\sigma| \leqq|\tau|$, then $|\sigma-i \tau| \leqq 2|\tau|$, so the first integral is bounded by

$$
\int_{-|\tau|}^{|\tau|} \exp \left\{-\frac{A \sigma^{2}}{4}|\tau|^{-\lambda / \gamma}+2 B|\tau|^{1 / \gamma}\right\} d \sigma \leqq C|\tau|^{\lambda /(2 \gamma)} \exp \left(2 B|\tau|^{1 / \gamma}\right) .
$$

If $|\sigma| \geqq|\tau|$, then $|\sigma+i \tau| \leqq 2|\sigma|$, so

$$
\begin{aligned}
& \int_{|\sigma| \geqq|\tau|} \exp \left\{-\frac{A \sigma^{2}}{2}|\sigma+i \tau|^{-\lambda / \gamma}+B|\tau||\sigma+i \tau|^{(1-\gamma) / \gamma}\right\} d \sigma \\
& \leqq 2 \int_{-\infty}^{\infty} \exp \left\{-\frac{A|\sigma|^{2-\lambda / \gamma}}{4}+2 B|\tau||\sigma|^{(1-\gamma) / \gamma}\right\} d \sigma .
\end{aligned}
$$


Put $\sigma=s|\tau|^{\gamma /(3 \gamma-\lambda-1)}$. Then the last integral is equal to

$$
\begin{aligned}
|\tau|^{\gamma /(3 \gamma-\lambda-1)} & \int_{0}^{\infty} \exp \left\{-|\tau|^{(2 \gamma-\lambda) /(3 \gamma-\lambda-1)}\left(-\frac{A s^{2-\lambda / \gamma}}{4}+2 B s^{(1-\gamma) / \gamma}\right)\right\} d s \\
& \leqq C|\tau|^{\gamma /(3 \gamma-\lambda-1)}|\tau|^{-(\gamma-\lambda / 2) /(3 \gamma-\lambda-1)} \exp \left(D|\tau|^{(2 \gamma-\lambda) /(3 \gamma-\lambda-1)}\right) \\
& =C|\tau|^{(\lambda / 2) /(3 \gamma-\lambda-1)} \exp \left(D|\tau|^{(2 \gamma-\lambda) /(3 \gamma-\lambda-1)}\right)
\end{aligned}
$$

with some $D>0$. Hence

$$
\begin{aligned}
\int_{-\infty}^{\infty}|\chi(\sigma-i \tau)| d \sigma \leqq & C\left\{|\tau|^{\lambda /(2 \gamma)} \exp \left(2 B|\tau|^{1 / \gamma}\right)\right. \\
& \left.+|\tau|^{(\lambda / 2) /(3 \gamma-\lambda-1)} \exp \left(D|\tau|^{(2 \gamma-\lambda) /(3 \gamma-\lambda-1)}\right)\right\} .
\end{aligned}
$$

The inequality $\lambda \geqq 2 \gamma-1$ implies that

$$
\frac{2 \gamma-\lambda}{3 \gamma-\lambda-1} \geqq \frac{1}{\gamma}
$$

so we may keep only the second term:

$$
\int_{-\infty}^{\infty}|\chi(\sigma-i \tau)| d \sigma \leqq C_{0}|\tau|^{(\lambda / 2) /(3 \gamma-\lambda-1)} \exp \left(D|\tau|^{(2 \gamma-\lambda) /(3 \gamma-\lambda-1)}\right) .
$$

Hence by (A.13)

Now,

$$
p(x) \leqq C_{0}|\tau|^{(\lambda / 2) /(3 \gamma-\lambda-1)} \exp \left(-x \tau+D|\tau|^{(2 \gamma-\lambda) /(3 \gamma-\lambda-1)}\right) .
$$

$$
\min _{\tau}\left(-x \tau+D|\tau|^{(2 \gamma-\lambda) /(3 \gamma-\lambda-1)}\right)=-D_{0}|x|^{(2 \gamma-\lambda) /(1-\gamma)},
$$

and it achieves at

so

$$
\tau=C_{1}|x|^{(3 \gamma-\lambda-1) /(1-\gamma)}
$$

$$
p(x) \leqq C_{2}|x|^{\lambda /(2(1-\gamma))} \exp \left(-D_{0}|x|^{(2 \gamma-\lambda) /(1-\gamma)}\right) \leqq C_{3} \exp \left(-\left(D_{0} / 2\right)|x|^{(2 \gamma-\lambda) /(1-\gamma)}\right),
$$

and Theorem A.1 is proved.

\section{Appendix B. Proof of Theorem 4.3}

For completeness we state three theorems, only one of which is needed for the purposes of this paper. We define

$$
S_{\alpha}(b)=\sum_{n=1}^{\infty}\left|r_{\alpha}(n)\right|^{2} \exp (-n / b)
$$

Theorem B.1 = 4.3. For all $\alpha$,

$$
\liminf _{b \rightarrow \infty}\left(b^{-1} S_{\alpha}(b)\right) \geqq \pi
$$

Theorem B.2. Except for an exceptional set of $\alpha$ of measure zero in $R^{2}$,

$$
S_{\alpha}(b)=\pi b+O\left(b^{(3 / 4)+\varepsilon}\right) \quad \text { as } b \rightarrow \infty .
$$


Theorem B.3. Suppose the vector $\alpha$ is rational, i.e. there exists an integer $Q$ such that

$$
2 Q \alpha_{1}=n_{1}, \quad 2 Q \alpha_{2}=n_{2}
$$

are integers and $\left(Q, n_{1}, n_{2}\right)$ have no common factor. Then

where

$$
S_{\alpha}(b)=(C / r(Q))(b / Q) \log (b / Q)+O(b) \quad \text { as } b \rightarrow \infty,
$$

$$
r(Q)=\prod_{p \mid Q}\left(1+p^{-1}\right)
$$

with the product taken over primes $p$ dividing $Q$, and

$C=3(Q$ even $), \quad C=4\left(Q\right.$ odd,$\left(n_{1}+n_{2}\right)$ even $), \quad C=2\left(Q\right.$ odd,$\left(n_{1}+n_{2}\right)$ odd $)$.

Theorems B.2 and B.3 give more precise estimates of $S_{\alpha}(b)$ than Theorem B.1, valid for some but not all $\alpha$. We give here only the proof of Theorem B.1. This proof can easily be extended to cover Theorem B.3, but Theorem B.2 requires a different approach.

The definitions (4.2) and (B.1) give

$$
S_{\alpha}(b)=\sum_{m m^{\prime}} e\left(\alpha \cdot\left(m-m^{\prime}\right)\right) \exp \left(-m^{2} / b\right),
$$

summed over integer vectors $\left(m, m^{\prime}\right)$ with

$$
m^{2}=m^{\prime 2} \text {. }
$$

To convert (B.8) into an unrestricted sum, we write

$$
m_{1}=(j k+h l) / 2, \quad m_{1}^{\prime}=(j k-h l) / 2, \quad m_{2}=(j l-h k) / 2, \quad m_{2}^{\prime}=(j l+h k) / 2,
$$

or conversely

$$
m_{1}+m^{\prime}{ }_{1}=j k, \quad m_{1}-m^{\prime}{ }_{1}=h l, \quad m_{2}+m^{\prime}{ }_{2}=j l, \quad-m_{2}+m_{2}^{\prime}=h k .
$$

If any four integers $(j, k, l, h)$ are given satisfying

$$
\begin{gathered}
h^{2}+j^{2} \neq 0, k^{2}+l^{2} \neq 0, \\
\text { either } j \equiv h \equiv 0, \quad \text { or } k \equiv l \equiv 0, \quad \text { or } j \equiv h \equiv k \equiv l \equiv 1(\bmod 2),
\end{gathered}
$$

then (B.10) defines uniquely the integer vectors $\left(m, m^{\prime}\right)$ with

$$
4 m^{2}=4 m^{\prime 2}=\left(j^{2}+h^{2}\right)\left(k^{2}+l^{2}\right) \neq 0 .
$$

But there may be many sets $(j, k, l, h)$ corresponding to one pair $\left(m, m^{\prime}\right)$.

The pair $\left(m, m^{\prime}\right)$ fixes uniquely the ratio

$$
f=(k / l)=\left(m_{1}+m^{\prime}{ }_{1}\right) /\left(m_{2}+m^{\prime}{ }_{2}\right)=\left(-m_{2}+m^{\prime}{ }_{2}\right) /\left(m_{1}-m^{\prime}{ }_{1}\right) \text {. }
$$

If one of the fractions (B.15) is indeterminate (zero/zero), the other fraction is determinate. In all cases the ratio $f$ is determinate, being either finite, zero or infinite. If $f$ is finite, we fix $k$ and $l$ by reducing the fraction to its lowest terms, thus

$$
(k, l)=1, \quad l>0 \text {. }
$$


If $f=0$ we take $k=0, l=1$, and if $f=\infty$ we take $k=1, l=0$. With these choices of $k$ and $l$,

$$
\begin{gathered}
m_{1}+{m^{\prime}}_{1} \equiv-m_{2}+m^{\prime}{ }_{2} \equiv 0(\bmod k), \\
m_{2}+m^{\prime}{ }_{2} \equiv m_{1}-m^{\prime}{ }_{1} \equiv 0(\bmod l),
\end{gathered}
$$

where congruence $(\bmod 0)$ means equality. The integers

$$
\begin{gathered}
j=\left(m_{1}+m^{\prime}{ }_{1}\right) / k=\left(m_{2}+m^{\prime}{ }_{2}\right) / l, \\
h=\left(m_{1}-m^{\prime}{ }_{1}\right) / l=\left(-m_{2}+m^{\prime}{ }_{2}\right) / k,
\end{gathered}
$$

are uniquely fixed in all cases. The mapping from $\left(m, m^{\prime}\right)$ satisfying (B.9), to $(j, k, l, h)$ satisfying (B.12), (B.13), (B.16) is one-to-one. It is more convenient to make the mapping one-to-two by dropping the condition $(l>0)$ from (B.16). Then

$$
S_{\alpha}(b)=(1 / 2) \sum_{k l j h} e\left(h\left(l \alpha_{1}-k \alpha_{2}\right)\right) \exp \left(-\left(k^{2}+l^{2}\right)\left(j^{2}+h^{2}\right) / 4 b\right),
$$

summed over all $(j, k, l, h)$ satisfying (B.12), (B.13) and

$$
(k, l)=1 .
$$

There are now two terms $(k= \pm 1, l=0)$ corresponding to $f$ infinite in (B.15).

When (B.22) holds, the possibilities allowed by (B.13) reduce to two. Therefore

$$
S_{\alpha}(b)=S_{e}+S_{o},
$$

where the terms with $j$ and $h$ even are

$$
S_{e}=(1 / 2) \sum_{k l}[F(w) F(0)-1],
$$

summed over integers $(k, l)$ satisfying (B.22), and the terms with $j$ and $h$ odd are

$$
S_{o}=(1 / 2) \sum_{k l} G(w) G(0),
$$

summed over odd integers $k$ and $l$ satisfying (B.22). The functions $(F, G)$ are defined by

$$
\sum_{x} \exp \left(-x^{2} / a\right) e(x t)=F(t) \text { or } G(t)
$$

where the sum is over integer $x$ for $F$ and over half-odd-integer $x$ for $G$. In (B.24), (B.25), (B.26) we have used the abbreviations

$$
w=2\left(l \alpha_{1}-k \alpha_{2}\right), \quad a=b\left(k^{2}+l^{2}\right)^{-1} .
$$

The $(-1)$ in (B.24) takes account of the fact that the term $(j=h=0)$ was omitted from (B.21). By the Poisson summation formula, (B.26) gives

$$
\begin{aligned}
& F(t)=(\pi a)^{1 / 2} \sum_{p} \exp \left(-\pi^{2} a(p+t)^{2}\right), \\
& G(t)=(\pi a)^{1 / 2} \sum_{p}(-1)^{p} \exp \left(-\pi^{2} a(p+t)^{2}\right) .
\end{aligned}
$$


According to (B.26), the functions $F$ and $G$ are periodic with periods 1 and 2 respectively,

$$
F(w+1)=F(w), \quad G(w+1)=-G(w) .
$$

To estimate the behavior of $S_{\alpha}(b)$ by (B.24) and (B.25), the essential requirement is to determine the distribution of the residues of $w$ given by (B.27), modulo 1 and modulo 2. For any real number $g$, let $[g]$ be the integer part and

the fractional part of $g$. Let

$$
\{g\}=g-[g]
$$

$$
\hat{g}=\min (\{g\}, 1-\{g\})
$$

be the distance of $g$ from the nearest integer, and (by an abuse of notation) let $(-1)^{g}$ denote the parity of the nearest integer to $g$; for half-integer $g=n+(1 / 2)$ we define $(-1)^{g}=1$. To estimate (B.23) we need separate estimates of $F(w)$ and $G(w)$ for large and small $a$. For $a \leqq 1,(\mathrm{~B} .26)$ gives

$$
\begin{aligned}
F(w) & \left.=1+O\left(\exp \left(-a^{-1}\right)\right), \quad G(w)=O\left(\exp (-4 a)^{-1}\right)\right) \\
(\partial F / \partial w) & =O\left(\exp \left(-a^{-1}\right)\right), \quad(\partial G / \partial w)=O\left(\exp \left(-(4 a)^{-1}\right)\right) \\
(\partial F / \partial a) & =O\left(a^{-2} \exp \left(-a^{-1}\right)\right), \quad(\partial G / \partial a)=O\left(a^{-2} \exp \left(-(4 a)^{-1}\right)\right) .
\end{aligned}
$$

For $a \geqq 1,($ B.28) and (B.29) give

$$
\begin{aligned}
F(w) & =(\pi a)^{1 / 2}\left[\exp \left(-\pi^{2} a \hat{w}^{2}\right)+O\left(\exp \left(-(\pi / 2)^{2} a\right)\right)\right] \\
G(w) & =(-1)^{w}(\pi a)^{1 / 2}\left[\exp \left(-\pi^{2} a \hat{w}^{2}\right)+O\left(\exp \left(-(\pi / 2)^{2} a\right)\right)\right], \\
(\partial(F, G) / \partial w) & =O\left(a^{3 / 2} \hat{w} \exp \left(-\pi^{2} a \hat{w}^{2}\right)\right) \\
(\partial(F, G) / \partial a) & =O\left(\left(a^{-1 / 2}+a^{1 / 2} \hat{w}^{2}\right) \exp \left(-\pi^{2} a \hat{w}^{2}\right)\right) .
\end{aligned}
$$

The constants implied in the $O$ notation in (B.33)-(B.39) are independent of $w$ and $a$.

We now proceed to prove Theorem B.1, assuming that the vector $\alpha$ is irrational. We return at the end to the case of rational $\alpha$. Let $u$ be any irrational number and let $(M / N)$ be one of the continued-fraction convergents to $u$. Such convergents exist with $N$ arbitrarily large and satisfy

$$
|\eta|<N^{-1}, \quad \eta=M-N u \text {. }
$$

Consider any $N$ successive fractional parts of multiples of $u$,

$$
F_{j}=\{j u\}, \quad j=L, L+1, \ldots, L+N-1 .
$$

Since $M$ and $N$ are coprime, the relation

$$
k \equiv M j(\bmod N), \quad 0 \leqq k<N,
$$

between integers $j$ and $k$ is one-to-one. Then (B.40) and (B.41) give

$$
F_{j}=\{(k / N)-(J \eta / N)-((j-J) \eta / N)\}, \quad J=L+(1 / 2)(N-1) .
$$

Therefore,

$$
\left|F_{j}-(k / N)-\bar{f}\right|<(1 / 2)|\eta|<(2 N)^{-1}, \quad k=0,1, \ldots, N-1,
$$


with $\bar{f}$ independent of $j$. That is to say, the numbers $F_{j}$ lie on the interval $[0,1]$ with great uniformity.

Since we are assuming that $\alpha$ is irrational, we may suppose without loss of generality that $\alpha_{2}$ is irrational. We write

$$
u=-2 \alpha_{2}, \quad v=2 \alpha_{1}, \quad w=k u+l v,
$$

in agreement with (B.27). Let $I$ be any interval of length $\lambda$ contained in $[0,1]$, and let $S$ be any set of $s$ consecutive integers. We wish to estimate the number $n(S, I)$ of integers $k$ in $S$ for which $\{w\}$ lies in $I$. Choose any convergent $(M / N)$ to $u$, and divide $S$ into $[s / N]$ subsets of $N$ consecutive integers plus one subset of less than $N$ integers. Within each subset, by (B.44), the $\{w\}$ lie within $(2 N)^{-1}$ of a uniformly spaced set of numbers with spacing exactly equal to $N^{-1}$. In the uniformly spaced set, at least $(\lambda N-2)$ lie within $I$ and distant from the ends of $I$ by at least $(2 N)^{-1}$. Therefore at least $(\lambda N-2)$ integers in each subset have $\{w\}$ in $I$, and all the subsets together give

$$
n(S, I)>((s / N)-1)(\lambda N-2) .
$$

A similar argument, using one additional subset to include the whole of $S$, gives

$$
n(S, I)<((s / N)+1)(\lambda N+2) .
$$

We take $N$ and $s$ large enough so that

Then (B.46) and (B.47) give

$$
s>N^{2}, \quad(s / N)>N>2 .
$$

$$
|n(S, I)-\lambda s|<(4 / N) s .
$$

Note that the bound on the right-hand side of (B.49) is independent of the position and the size of $I$.

Now consider a square block $B$ of $\left(s^{2}\right)$ pairs of integers $(k, l)$ with $k$ in $S_{1}$ and $l$ in $S_{2}$, where $S_{1}$ and $S_{2}$ are sets of $s$ consecutive integers. We assume that $B$ does not include the pair $(0,0)$. We wish to estimate the number $C(B, I)$ of pairs in $B$ with $\{w\}$ in $I$ and satisfying (B.22). We use the Möbius inversion formula

$$
\sum_{(k, l)=1} f(k, l)=\sum_{d k l} \mu(d) f(d k, d l)
$$

valid for any function $f$ with compact support in $(k, l)$. Provided that the support of $f$ does not include $(0,0)$, the sum on the right of (B.50) contains only a finite number of terms. Applying (B.50) to the block $B$, we find

$$
\left.C(B, I)=\sum_{d} \mu(d) n\left(S_{1} / d\right),(I / d)\right) n\left(\left(S_{2} / d\right), I_{0}\right),
$$

where $(S / d)$ means the set of integers $k$ such that $(d k)$ belongs to $S,(I / d)$ means the set of real numbers $\{w\}$ such that $\{d w\}$ belongs to $I$, and $I_{0}$ is the whole interval $[0,1]$. If $I$ is an interval of length $\lambda$, then $(I / d)$ is a sum of $d$ intervals with total length $\lambda$. Thus (B.49) implies

while

$$
\left|n\left(\left(S_{1} / d\right),(I / d)\right)-(\lambda s / d)\right|<(4 / N) s d,
$$

$$
\left|n\left(\left(S_{2} / d\right), I_{0}\right)-(s / d)\right|<1 \text {. }
$$


We divide the sum (B.51) into two parts. The terms with $d<D$ are estimated using (B.52) and (B.53), the terms with $D<d<s$ using

$$
n((S / d), I)<2(s / d) \text {. }
$$

Terms in (B.51) with $d>s$ are zero since $(0,0)$ is not in $B$. Since

$$
\sum_{1}^{\infty} d^{-2} \mu(d)=\left(6 / \pi^{2}\right)
$$

the estimates (B.52), (B.53), (B.54) applied to (B.51) give

$$
C(B, I)=\left(6 / \pi^{2}\right) \lambda s^{2}+O\left(s^{2}\left(D N^{-1}+D^{-1}\right)\right) .
$$

We choose $D=N^{1 / 2}$ and obtain

$$
C(B, I)=\left(6 / \pi^{2}\right) \lambda s^{2}+O\left(s^{2} N^{-1 / 2}\right) .
$$

The meaning of (B.57) is that in every block $B$ the values of $\{w\}$ with $(k, l)$ satisfying (B.22) are distributed uniformly over the interval $[0,1]$.

We apply the estimate (B.57) to the sums (B.24), (B.25) with $F(w), G(w)$ given by (B.28), (B.29). The sums over $(k, l)$ are divided into boxes $B$ with size $s^{2}$, assumed to be small compared with $b$. Within each box, the values of $a$ given by (B.27) vary only over an interval of length

$$
\Delta a=s a(a / b)^{1 / 2},
$$

and the values of $\{w\}$ are distributed according to (B.57). We choose two integers $\mu$ and $M$, such that

$$
\delta=(\mu / M)
$$

is small compared with unity. We divide $S_{e}$ into two parts,

$$
S_{e}=S_{e 1}+S_{e 2},
$$

where $S_{e 1}$ is the sum of terms in (B.24) with

$$
\hat{w}>\delta,
$$

and $S_{e 2}$ is the remainder. Similarly, (B.25) is divided. We estimate separately the three contributions $S_{e 1}, S_{o 1}$, and $S_{2}=S_{e 2}+S_{o 2}$ to (B.23). We shall find that when $\delta$ is small and $b$ large, $S_{e 1}$ is asymptotically equal to the desired bound $\pi b, S_{o 1}$ is small, and $S_{2}$ is either positive or small.

To estimate $S_{e 1}$, we apply (B.57) to the $(M-2 \mu)$ intervals $[(m / M),((m+1) / M)]$, $m=\mu, \ldots, M-\mu-1$. The result is

with

$$
S_{e 1}=\sum_{k l, B} \int_{\delta}^{1-\delta} d w\left[\left(6 / \pi^{2}\right) f(a, w)+R(a, w)\right],
$$

$$
f(a, w)=(1 / 2)(F(w) F(0)-1),
$$

and the remainder term of order

$$
R(a, w)=O\left(M N^{-1 / 2} f\right)+O\left(M^{-1}(\partial f / \partial w)\right)+O((\Delta a)(\partial f / \partial a)) .
$$

After inserting the estimates (B.33)-(B.39) into (B.64), we can convert the summation over $(k, l)$ into an integration over $a$ using (B.27). Thus

$$
\sum_{k l} \text { becomes }(\pi b) \int_{0}^{\infty} a^{-2} d a
$$


and (B.62) becomes

with

$$
S_{e 1}=\pi b \int_{\delta}^{1-\delta} d w[J(w)+R]
$$

$$
\begin{aligned}
J(w) & =\int_{0}^{\infty} a^{-2} d a\left(6 / \pi^{2}\right) f(a, w), \\
R & =O\left(M N^{-1 / 2}+M^{-1}+s b^{-1 / 2}\right) K(\delta), \\
K(\delta) & =\int_{1}^{\infty} a^{-1 / 2} d a \exp \left(-\pi^{2} a \delta^{2}\right)+\int_{0}^{1} a^{-5 / 2} d a \exp \left(-a^{-1}\right) .
\end{aligned}
$$

By (B.26),

$$
\int_{0}^{1} F(w) d w=1, \quad \int_{0}^{\infty} a^{-2}(F(0)-1) d a=\sum_{x \neq 0} x^{-2}=\left(\pi^{2} / 3\right),
$$

so that (B.67) gives

$$
\int_{0}^{1} J(w) d w=1
$$

From (B.71), (B.33) and (B.36) it follows that

$$
\int_{\delta}^{1-\delta} J(w) d w=1+O(\delta|\log \delta|) .
$$

The integrals on the right of (B.69) are convergent and give contributions of order $\delta^{-1}$ at most. Therefore (B.66), (B.68) and (B.72) together give

$$
S_{e 1}=\pi b\left(1+O(\delta|\log \delta|)+O\left(\delta^{-1}\left(M N^{-1 / 2}+M^{-1}+s b^{-1 / 2}\right)\right)\right) .
$$

After choosing $\delta$ as small as we please, we may choose the other parameters in turn,

$$
M=\delta^{-2}, \quad N>M^{4}, \quad s=N^{2}, \quad b>N^{4} \delta^{-4},
$$

with $N$ a denominator of a convergent to $u$ according to (B.40). Then (B.73) reduces to

$$
S_{e 1}=(\pi b)(1+O(\delta|\log \delta|)) .
$$

The estimation of $S_{o 1}$ proceeds in exactly the same way, except that $G(w)$ is periodic with period 2 instead of 1 , and by (B.30)

$$
\int_{0}^{2} G(w) d w=0
$$

instead of (B.70). Therefore the 1 is missing in the analog of (B.73), and we have instead of (B.75)

$$
S_{o 1}=(\pi b) O(\delta|\log \delta|) .
$$

Lastly we estimate $S_{2}$, the part of the sums (B.24) and (B.25) that have

$$
\hat{w}<\delta \text {. }
$$

We divide the sums into boxes $B$ of size $s^{2}$ as before. Within each box, the fraction of pairs $(k, l)$ satisfying (B.22) and (B.78) is by (B.57)

$$
f=\left(6 / \pi^{2}\right) 2 \delta+O\left(N^{-1 / 2}\right)=O(\delta),
$$


when $N$ is chosen to satisfy (B.74). We are concerned only with finding a lower bound for $S_{2}$, and so we may drop any positive terms from the summand. By (B.26), (B.28) and (B.29),

$$
F(w)>|G(w)|>0,
$$

so that the summand in $S_{2}$ is everywhere greater than $(-1)$. Also, for $a \leqq 1$, the summand is by (B.33) uniformly majorized by

$$
O\left(\exp \left(-(4 a)^{-1}\right)\right) \text {. }
$$

Converting the sums as before to integrals over $a$, we find from (B.79), (B.80) and (B.81),

$$
S_{2}>-f \pi b(1 / 2)\left(\int_{1}^{\infty} a^{-2} d a+O\left(\int_{0}^{1} a^{-2} \exp \left(-(4 a)^{-1}\right) d a\right)\right) .
$$

The integrals are convergent, and so (B.79) and (B.82) imply

with an absolute constant $C$.

$$
S_{2}>-C b \delta \text {, }
$$

It remains only to assemble (B.75), (B.77) and (B.83) and obtain

$$
b^{-1} S_{\alpha}(b)>\pi-C \delta|\log \delta|,
$$

with another constant $C$, provided that $b$ is large enough to satisfy (B.74). Therefore the left side of (B.2) is bounded by the right side of (B.84). But the left side of (B.2) is independent of $\delta$. Letting $\delta$ tend to zero, the right side of (B.84) becomes $\pi$, and Theorem B.1 is proved for $\alpha$ irrational.

Suppose finally that $\alpha$ is rational, i.e. there exists an integer $Q$ satisfying (B.4). Then by (B.27), $w$ is an integer multiple of $Q^{-1}$. Accordingly, (B.24) and (B.25) imply

$$
\begin{aligned}
& S_{e}=(1 / 2) \sum_{k l, i}\left[(F(0))^{2}-1\right]+(1 / 2) \sum_{k l, n}[F(\hat{w}) F(0)-1], \\
& S_{o}=(1 / 2) \sum_{k l, i}(-1)^{w}(G(0))^{2}+(1 / 2) \sum_{k l, n}(-1)^{w} G(\hat{w}) G(0),
\end{aligned}
$$

where the sums labeled $i$ are over the terms with integer $w$ and the terms labeled $n$ are over non-integer $w$ with

$$
\hat{w} \geqq Q^{-1}
$$

According to (B.33), (B.36) and (B.37), the non-integer terms are majorized by

$$
\sum_{k l} O\left(\min \left(\exp \left(-(4 a)^{-1}\right), a \exp \left(-(\pi / Q)^{2} a\right)\right)\right),
$$

with the sum extended over all integers $(k, l)$, whether they satisfy (B.22) or not. When $b$ is large, the sum in (B.88) becomes an integral over $a$ according to (B.65). The integral converges at large and small $a$, so that (B.88) is $O(b)$ as $b \rightarrow \infty$.

It remains to estimate the sums in (B.85) and (B.86) with integer $w$. These sums give

$$
S_{\alpha}(b)=(1 / 2) \sum_{k l}\left(\Delta\left((F(0))^{2}-1\right)+\left(\Delta_{1}-\Delta_{2}\right)(G(0))^{2}\right)+O(b),
$$

with the sums extended over all pairs $(k, l)$, where $\Delta$ is the density of integer pairs $(k, l)$ satisfying $($ B.22) and

$$
\ln n_{1}-k n_{2} \equiv 0(\bmod Q)
$$


while $\left(\Delta_{1}, \Delta_{2}\right)$ are the densities of pairs with both $k$ and $l$ odd satisfying (B.22) and

$$
\ln _{1}-k n_{2} \equiv(0, Q)(\bmod 2 Q) \text {. }
$$

The sums in (B.89) can be estimated with an error of order $b$ by using (B.33), (B.36) and (B.37). To this accuracy, the terms with $a \leqq 1$ are negligible, and the terms with $a>1$ can be approximated by taking

$$
(F(0))^{2}-1=(G(0))^{2}=\pi a,
$$

so that

with

$$
S_{\alpha}(b)=(D \pi b / 2) \sum_{a>1}\left(k^{2}+l^{2}\right)^{-1}+O(b),
$$

$$
D=\Delta+\Delta_{1}-\Delta_{2} .
$$

When $b$ is large compared with $Q$, the sum in (B.93) can be approximated by an integral as before. With an error of order unity as $b \rightarrow \infty$,

$$
\sum_{a>1}\left(k^{2}+l^{2}\right)^{-1}=\pi \int_{1}^{A} a^{-1} d a=\pi \log A .
$$

The upper limit of integration in (B.95) is according to (B.27)

$$
A=b\left(\min \left(k^{2}+l^{2}\right)\right)^{-1}=(b / Q),
$$

neglecting a geometrical factor of order unity, since the smallest value of $\left(k^{2}+l^{2}\right)$ satisfying (B.90) will be of order $Q$. Thus (B.93) becomes

$$
S_{\alpha}(b)=\left(D \pi^{2} b / 2\right) \log (b / Q)+O(b) .
$$

It is not difficult to calculate $D$ explicitly, with the result stated in Theorem B.3. For the proof of Theorem B.1, it is sufficient to observe that $D$ is a positive number depending only on $Q, n_{1}$ and $n_{2}$ and independent of $b$. The fact that $D$ is positive follows immediately from the definitions (B.90), (B.91) and (B.94). Then (B.97) implies that for rational $\alpha$

$$
\lim _{b \rightarrow \infty}\left(b^{-1} S_{\alpha}(b)\right)=\infty,
$$

and the proof of Theorem B.1 is complete.

Acknowledgements. The authors thank E. Bombieri and D.R. Heath-Brown for a copy of the paper [H-B] and for discussions of this paper. They thank also the anonymous referee for useful remarks. The first author is grateful to the Institute for Advanced Study, Princeton, where part of this work was done, for financial support of his visit to the Institute. P.M.B., Z.C. and J.L.L. were partly supported by National Science Foundation Grant DMR 89-18903.

\section{References}

[B] Berry, M.V.: Semiclassical theory of spectral rigidity. Proc. Roy. Soc. London, Ser. A 400, 229-251 (1985)

[Be] Beck, J.: Probabilistic Diophantine approximations. Preprint, Rutgers University, 1992

[B11] Bleher, P.M.: Quasiclassical expansion and the problem of quantum chaos. Lect. Notes in Math. 1469, 60-89 (1991)

[B12] Bleher, P.M.: On the distribution of the number of lattice points inside a family of convex ovals. Duke Math. J. 67, 461-481 (1992) 
[B13] Bleher, P.M.: Distribution of the error term in the Weyl asymptotics for the Laplace operator on a two-dimensional torus and related lattice problems. Preprint, Tel Aviv University, 1992 (to appear in Duke Math. Journ.)

[CCG] Casati, G., Chirikov, B.V., Guarneri, I.: Energy-level statistics of integrable quantum systems. Phys. Rev. Lett. 54, 1350-1353 (1985)

[CdV] Colin de Verdière, Y.: Nombre de points entiers dans une famille homothétique de domaines de $\mathbf{R}^{n}$. Ann. Scient. Éc. Norm. Sup., $4^{e}$ série, 10, 559-576 (1977)

[CFS] Cornfeld, I.P., Fomin, S.V., Sinai, Ya.G.: Ergodic Theory. New York: Springer 1982

[CK] Corradi, K., Katai, I.: A comment on K.S. Gangadharan's paper entitled "Two classical lattice point problems." Magyar Tud. Akad., Mat. Fiz. Oszt. Közl. 17, 89-97 (1967)

[CL] Cheng, Z., Lebowitz, J.L.: Statistics of energy levels in integrable quantum systems. Phys. Rev. A44, R3399-R3402 (1991)

[G] Grosswald, E.: Representations of integers as sums of squares. New York e.a.: SpringerVerlag 1985

[Haf] Hafner, J.L.: New Omega theorems for two classical lattice point problems. Invent. Math. 63, 181-186 (1981)

[H1] Hardy, G.H.: The average order of the arithmetical functions $P(x)$ and $\Delta(x)$. Proc. London Math. Soc. 15, 192-213 (1916)

[H2] Hardy, G.H.: On Dirichlet's divisor problem. Proc. London Math. Soc. 15, 1-25 (1916)

[HW] Hardy, G.H., Wright, E.M.: An introduction to the theory of numbers. (4th ed.) Oxford 1960

[H-B] Heath-Brown, D.R.: The distribution and moments of the error term in the Dirichlet divisor problem. Preprint, Oxford University (to appear in Acta Arithmetica)

[Hux] Huxley, M.N.: Exponential sums and lattice points. II. Preprint, University of Wales College of Cardiff, 1992

[IM] Iwaniec, H., Mozzochi, C.J.: On the divisor and circle problems. J. Number Theory 29, 60-93 (1988)

[I] Ivić, A.: The Riemann Zeta-function. New York: Wiley 1985

[K] Kendall, D.G.: On the number of lattice points inside a random oval. Quart. J. of Math. (Oxford) 19, 1-26 (1948)

[S] Sinai, Ya.G.: Mathematical problems in the theory of quantum chaos. Lect. Notes in Math. 1469, 41-59 (1991)

[T] Titchmarsh, E.C.: The theory of the Riemann Zeta-function. (2nd ed.) Oxford 1986

[Ts] Tsang, K.-M.: Higher power moments of $\Delta(x), E(x)$ and $P(x)$ (to appear)

Communicated by T. Spencer 
